\title{
Article \\ Time-Interval-Varying Optimal Power Dispatch Strategy Based on Net Load Time-Series Characteristics
}

\author{
Shubo Hu ${ }^{1,2} \oplus$, Zhengnan Gao ${ }^{2}$, Jing Wu ${ }^{1}$, Yangyang Ge ${ }^{1}$, Jiajue $\mathrm{Li}^{1}$, Lianyong Zhang ${ }^{3}$, Jinsong Liu ${ }^{1}$ \\ and Hui Sun $2, * \mathbb{D}$
}

Citation: Hu, S.; Gao, Z.; Wu, J.; Ge, Y.; Li, J.; Zhang, L.; Liu, J.; Sun, H. Time-Interval-Varying Optimal Power Dispatch Strategy Based on Net Load Time-Series Characteristics. Energies 2022, 15, 1582. https:// doi.org/10.3390/en15041582

Academic Editors: Nicu Bizon and Marco Pau

Received: 7 January 2022

Accepted: 14 February 2022

Published: 21 February 2022

Publisher's Note: MDPI stays neutral with regard to jurisdictional claims in published maps and institutional affiliations.

Copyright: (C) 2022 by the authors. Licensee MDPI, Basel, Switzerland. This article is an open access article distributed under the terms and conditions of the Creative Commons Attribution (CC BY) license (https:// creativecommons.org/licenses/by/ $4.0 /)$
1 Electric Power Research Institute of State Grid Liaoning Electric Power Co., Ltd., Shenyang 110000, China; shubo_hu@dlut.edu.cn (S.H.); wj_ldk@ln.sgcc.com.cn (J.W.); gyy_ldk@ln.sgcc.com.cn (Y.G.); lijj_ldk@ln.sgcc.com.cn (J.L.); ljs_ldk@ln.sgcc.com.cn (J.L.)

2 School of Electrical Engineering, Dalian University of Technology, Dalian 116024, China; dut_nan@yeah.net 3 Inner Mongolia EHV Power Supply Bureau, Inner Mongolia Power (Group) Co., Ltd., Hohhot 010080, China; yifanfengshun1983@163.com

* Correspondence: sunhui_ee@163.com; Tel.: +86-13504247118

\begin{abstract}
In China, with the increasing permeability of wind power, the power supply capacity is enough overall, but has shortage in partial-time. During peak hours, the capability of wind power consumption is poor and the power balance becomes more difficult. In order to maximize the utilization of wind power, the net loads are chosen as the response objectives, which contain significant uncertainties and have no probabilistic distribution characteristics. Under the traditional day-ahead power dispatch mode with fixed length time intervals and in the regions with insufficient hydroelectricity, the thermal generators take charge of the peak-load shaving. The frequent adjustments of thermal power output affect the system operation safety, economic benefits, and environmental benefits. Thus, a time-interval-varying optimal power dispatch strategy based on net load time-series characteristics is proposed in this paper. The net loads respond differently to intervals. The length of each time interval is determined based on the net load time-series characteristics analyzed by random matrix theory. The dispatch mode in each time interval is determined according to the characteristic quantification index calculated by the empirical modal decomposition and sample entropy. The proposed strategy and method can extend the continuous and stable operation time of the thermal generators, reduce the coal consumption caused by the ramping operation, and improve the safety, stability, and economy of the system. Furthermore, the proposed dispatch mode is environmentally friendly with reduced environmental cost and increased carbon credits. An actual provincial power grid in northeast China is taken as the example to verify the rationality and effectiveness of the proposed method and strategy.
\end{abstract}

Keywords: carbon credit; net load; power system optimization; renewable power consumption; time-interval-varying optimal power dispatch; wind power

\section{Induction}

With the rapid development of wind power and the transformation of energy structure, the new energy development targets provided in the National Energy Administration '14th five-year' power planning work are beginning to be met in China. The targets contain the improvement of power safety guarantee capability, promotion of structural reform on power supply side, development of the traditional power generators, and the improvement of overall power system efficiency [1]. In the areas with poor water resources, the high penetration of wind power access to the power grid will cause difficulty in the power balance control, owing to the difficulty in wind power consumption during peak hours [2].

In the traditional day-ahead power dispatch with wind power, there are strong uncertainties in the system. Under the fixed number and length time-scale power dispatch mode, the power generators need to respond to the load demands and uncertainties every 
$15 \mathrm{~min}$, half-hour, or $1 \mathrm{~h}[3,4]$. However, the adjustments of thermal power output concern increasing or decreasing air inflow, coal consumption, and many other mechanical operations. Thus, the frequent changes of the thermal power output cause adjustments of boiler steam pressure and temperature, which bring about mechanical loss, thermal fatigue, and vibration fatigue [5,6]. Meanwhile, carbon emissions are an important concern in the power system operation due to the threats of global warming [7]. The concept of carbon neutrality was first put forward in the Seventy-Fifth United Nations General Assembly in September 2020. The report states that the carbon dioxide emissions will reach a peak value in 2030 and carbon neutrality will be realized in 2060 [8]. One ton of carbon dioxide emission avoided or reduced can obtain one carbon credit, which can be traded between governments and enterprises. The carbon dioxide reduction capacity has become one of the assessment factors of an enterprise or a government. The traditional power dispatch modes are not suitable for today's power system energy structure and power balance demands. In order to improve the renewable energy response capability and reduce the emissions produced by traditional thermal generators, experts and scholars have made numerous studies.

With high proportion wind power connected to the power grid, the system contains strong uncertainties. In order to ensure the response ability of the generators, the ramping operation capacity and time-scale cooperation are of great importance [9]. In [10], the uncertainties of renewable power are considered in two time scales, day-ahead and intraday. Further, in [11], considering the operation characteristics of flexible loads, conventional generators and wind power, a coordinated dispatch strategy among three time scales: dayahead, intra-day, and real time, is proposed. In [12], the authors state that the shorter the time interval, the more accurate the renewable energy information is. On this basis, in [13], the ramping capacity for different time scales is studied and provides the generator ramping constraints in a subhour time scale to improve the response ability of the system. However, from the time-scale perspective, most of the research combines the day-ahead, intra-day, and real time and further to determine the dispatch strategy, in which the time length in each time scale is still the same. There are fewer studies on the time division according to the net load data adaptively and the existing methods have subjective factors. An objective and flexible dispatch time-interval division method is urgently needed.

In addition, due to the uncertainties of wind power and convention loads, the net loads have strong uncertainties and nonprobability distribution characteristics. Thus, from the perspective of data information, sample entropy (SampEn) can be used to analyze the complexities [14]. However, the research in this paper has some limits and analysis results are easily influenced by noise. In [15], empirical mode decomposition (EMD) and fuzzy entropy are combined to analyze the complexities of time series, which can extract the local features, but are not suitable to the time series with strong volatilities. In $[16,17]$, the timeseries analysis methods, such as the Markov chain model, are utilized to conduct real-time monitoring of time-series uncertainties. This research has provided methods and theories in net load characteristic analysis. However, the time-series characteristic quantification index calculation and the subsequent division need further studies. Currently, big data technology has been applied to power system analysis. As a data analysis technology, the random matrix theory (RMT) $[18,19]$ has been utilized in static stability analysis [20], transient stability analysis [21], fault identification [22], among others. The data dependency [23] and randomness [24] can be analyzed by RMT. Thus, it can be seen from these studies that RMT can recognize the changing volatility and stability of data.

With the development of power systems, data information has been an important influencing factor. However, how to apply the data information to the power system's optimal operation reasonably and efficiently is a technological difficulty. Especially under the target of zero wind curtailment, net loads have strong uncertainties. A new flexible and adaptable dispatch strategy-making method is urgently needed and we propose new methods aiming at these technological gaps. In this paper, we improve the traditional power dispatch mode. Facing a high proportion of wind power, the net loads are the 
response objectives that are used to achieve zero wind curtailment. The RMT is used to analyze the net load stability and assist with the time interval division. The EMD is used to extract the overall changing trend and SampEn is used to calculate the complexity of each net load subsequence. The characteristic quantification index is calculated by the EMD and SampEn. Further, a time-interval-varying optimal power dispatch model is built, which can achieve the continuous and stable operation of thermal generators and the participation improvement of energy storage systems. An actual provincial power grid is developed to prove the safety, stability, economy, and environmental friendliness.

\section{Time-Series Characteristics Analysis of Net Loads Based on RMT}

The random matrix is composed of random variables. When the number of the matrix rows and columns approach infinity and the ratio of row number to column number is constant, the empirical spectral distribution (ESD) is convergent to the limiting spectral distribution (LSD). The ESD can be formulated as follows:

$$
F^{\mathrm{A}}(x)=\frac{1}{N} \sum_{i=1}^{N} \phi\left(\lambda_{i}^{\mathrm{A}} \leq x\right)
$$

where $\mathbf{A}$ is a $N \times N$ random matrix; $\lambda_{i}^{\mathrm{A}}$ is the eigenvalue of $\mathbf{A} ; i=1,2, \ldots, N ; F^{\mathrm{A}}(x)$ is the probability of the eigenvalue satisfying the constraints of LSD.

\subsection{Monocyclic Theorem and the Calculation of the Mean Spectral Radius (MSR)}

In this paper, the monocyclic theorem is chosen as the LSD [25]. A non-Hermitian matrix formulated as $\tilde{X}=\left\{x_{i, j}\right\}_{N_{\mathrm{W}} \times T_{\mathrm{W}}}$ is built, in which all the elements are the random variables with independent identical distributions. In this matrix, the expectation is 0 and the variance is 1 . For the matrix $\tilde{X}_{i}(i=1, \ldots, L)$ that contains multiple non-Hermitian matrixes, their matrix products should be calculated as follows:

$$
\hat{Z}=\prod_{i=1}^{L} \tilde{X}_{u, i}
$$

where $\tilde{X}_{u, i} \in C^{N_{W} \times N_{W}}$ is the singular value matrix of $\tilde{X}_{i}$ and $C$ is the set of the complex number; $\tilde{Z}=\left\{\tilde{z}_{i, j}\right\}_{N_{\mathrm{W}} \times N_{\mathrm{W}}}$ is the standardization of $\hat{Z}$, where $E\left(\tilde{z}_{i, j}\right)=0$ and $E\left(\left|\tilde{z}_{i, j}\right|_{2}\right)=1 / N_{\mathrm{W}}$. When $N_{\mathrm{W}}, T_{\mathrm{W}} \rightarrow \infty$ and $c=N_{\mathrm{W}} / T_{\mathrm{W}} \in(0,1]$ is constant, the eigenvalues of $\tilde{Z}$ are distributed between two loops of the monocyclic theorem complex plane [23]. The corresponding probability density function can be formulated as follows:

$$
f(\lambda)=\left\{\begin{array}{l}
\frac{1}{\pi c L}|\lambda|^{\frac{2}{L}-2},(1-c)^{\frac{L}{2}} \leq|\lambda| \leq 1 \\
0, \text { others }
\end{array}\right.
$$

where the radius of the inner loop is $(1-c)^{L / 2}$ and the radius of the outer loop is 1 . The inner and outer loop distributed figure of the monocyclic theorem is shown in Figure 1.

The MSR [21] is a common statistic of linear eigenvalue and often used in the RMT to describe the distribution of the eigenvalue. According to the monocyclic theorem, MSR is the distance of an eigenvalue to the origin in the complex plane, which can be shown in Equation (4):

$$
K_{M S R}=\frac{1}{N} \sum_{n=1}^{N}\left|\lambda_{n}\right|, n=1,2, \ldots, N
$$

where the $K_{M S R}$ is the MSR and $\lambda_{n}$ is the $n$th eigenvalue. 


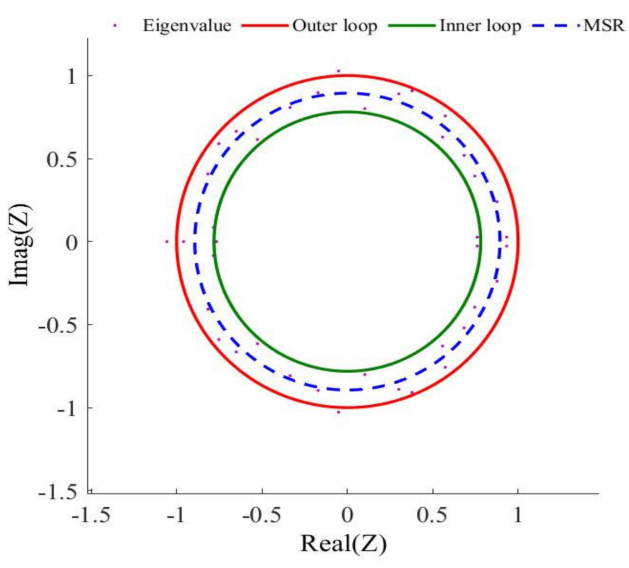

Figure 1. The inner and outer loop distributed figure of the monocyclic theorem.

\subsection{Time Interval Division Method of Net Load}

Data fluctuation characteristic can be analyzed by RMT. The time series of conventional load can be shown as $\{L(t)\}=\{l(1), l(2), \ldots, l(T)\}$ and the time series of uncertainty power generation can be expressed as $\{W(t)\}=\{w(1), w(2), \ldots, w(t)\}$; thus, the net load time series can be shown as $\{N L(t)\}=\{n l(1), n l(2), \ldots, n l(T)\}$ where $n l(t)=l(t)-w(t)$ and $\mathrm{T}$ is the total number of time intervals. The data of net load are influenced by the weather, date, social conditions, and many other factors. Thus, the data contain strong uncertainties, owing to which the high signal-to-noise ratio noise is selected to reduce the effects of net load fluctuation within a narrow range. The net load matrix with high signal-to-noise ratio can be formulated as Equation (5):

$$
N L^{\mathrm{H}}(i)=N L(i)+k^{\mathrm{H}} \times N(i)
$$

where the $N L^{\mathrm{H}}(i) \in C^{N_{\mathrm{W}} \times T_{\mathrm{W}}}$ is the matrix with high signal-to-noise ratio; $N L(i) \in C^{N_{\mathrm{W}} \times T_{\mathrm{W}}}$ is the net load matrix; $k^{\mathrm{H}}$ is the high signal-to-noise ratio; $N(i) \in C^{N_{W} \times T_{W}}$ is the noise matrix satisfying the standard normal distribution; and $N_{\mathrm{W}} \times T_{\mathrm{W}}$ is the size of sliding time window utilized to generate the net load matrix.

The MSR shows positive correlation with the data stability. The time division method of the net load time series based on high signal-to-noise ratio matrix is as follows: Firstly, calculate the stable value $w$ and the stability threshold $\delta_{\mathrm{MSR}}$. When the variation amplitude of MSR is larger than $\delta_{\mathrm{MSR}}$, the data stability starts to change. Secondly, look for points $A_{i}$, which are the initial dropping points of MSR from the stable value $w . A_{i}$ are the first kind of subsection points. Thirdly, if the MSR after $A_{i}$ cannot back up to $w \pm \delta_{\mathrm{MSR}}$, the corresponding time intervals are regarded as fluctuation data segments until the MSR back up to $w \pm \delta_{\text {MSR }}$. At this time, all the data in the data window are stable and the first point that begins to stabilize is the key point. These kinds of points are the minimum points before MSR backs up to stability range, and they are called the second kind of subsection points and expressed as $B_{i}$.

The schematic diagram of net load time division based on MSR is shown in Figure 2 and the flow chart is shown in Figure 3. 


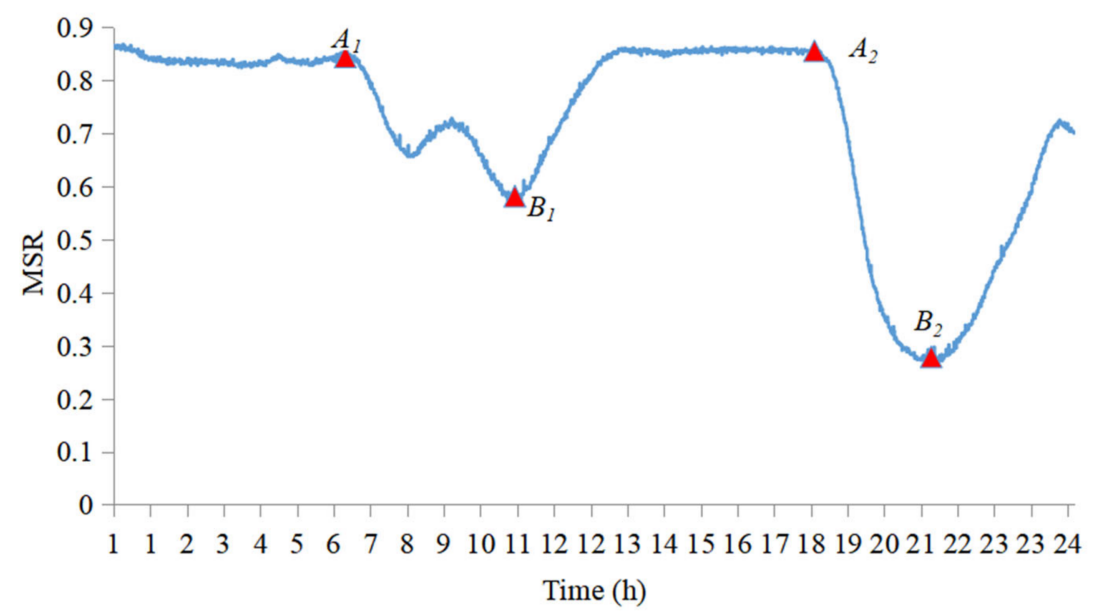

Figure 2. Schematic diagram of net load time division based on MSR.

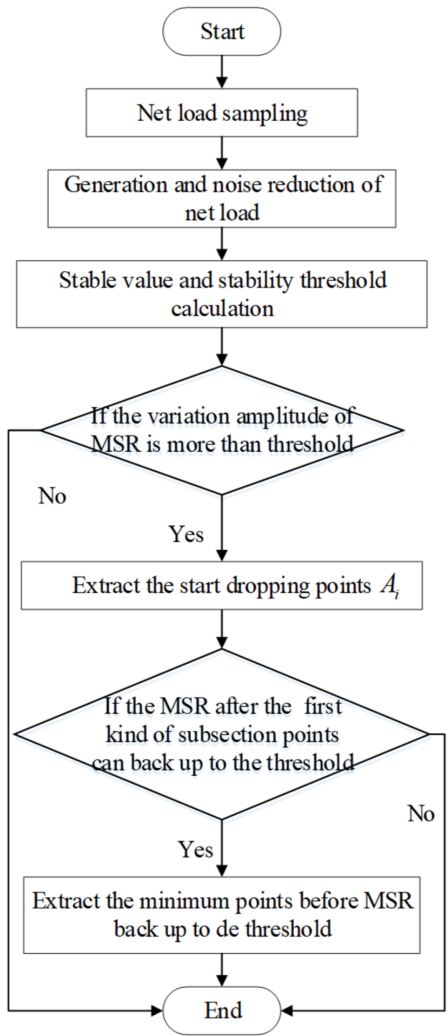

Figure 3. Flow chart of dispatch time division based on RMT.

\section{Quantification of Net Load Time-Series Characteristics}

\subsection{Numerical Characteristic Analysis Based on EMD}

The EMD is an adaptive signal time-frequency processing method, which is suitable for nonlinear and nonstationary signal analysis. In EMD, the original signal is decomposed into many narrow-band components and each component is called an intrinsic mode function (IMF). The calculation steps of EMD follow:

(1) Extract the maximum points and their fitting envelope curve $e_{\mathrm{up}}(t)$ of the original time series $n l(t)$;

(2) Extract the minimum points and their fitting envelope curve $e_{\text {low }}(t)$; 
(3) Calculate the average value $\bar{e}(t)$ of the up- and low-fitting envelope curves; which can be calculated as

$$
\bar{e}(t)=\frac{e_{\mathrm{up}}(t)+e_{\mathrm{low}}(t)}{2}
$$

(4) Update the net load time series with the equation $n l^{\prime}(t)=n l(t)-\bar{e}(t)$, and repeat Step (1) until the $n l^{\prime}(t)$ satisfies the constraints of IMF. The IMF has two constraints, the first is the numerical difference between the extreme points number and zero crossing points number, which is not more than 1 ; the second is that at any point the average value of the up- and low-fitting envelope curves is 0 . The $n l^{\prime}{ }_{1}(t)$ is the first IMF component that contains the highest frequency components.

(5) Extract the component $n l^{\prime}{ }_{1}(t)$ of IMF from the original signal $n l(t)$, and the remaining components are formulated as $r l_{1}(t)=n l(t)-n l_{1}^{\prime}(t)$.

(6) Set the remaining components $r l_{1}(t)$ as the new original signal and repeat the steps above. The other components of IMF and a margin can be achieved, which can be shown as follows:

$$
\left\{\begin{array}{c}
r l_{1}(t)-n l^{\prime}{ }_{2}(t)=r l_{2}(t) \\
r l_{2}(t)-n l_{3}{ }_{3}(t)=r l_{3}(t) \\
r l_{3}(t)-n l^{\prime}{ }_{4}(t)=r l_{4}(t) \\
\vdots \\
r l_{N-1}(t)-n l^{\prime}{ }_{N}(t)=r l_{N}(t)
\end{array}\right.
$$

Thus, the original signal can be formulated as $n l(t)=\sum_{i=1}^{N} n l^{\prime}{ }_{i}(t)+r l_{N}(t)$. When the $N$ th component $n l^{\prime}{ }_{N}(t)$ or its margin $r l_{N}$ is less than the presetting value, the decomposition process of EMD is finished. Otherwise, when the margin is a monotone function or a constant, the decomposition process of EMD is finished as well.

The curve of net loads can reflect their changing tendency and the changing direction changes after the extreme points. Figure 4 shows the original net loads of a provincial power grid in northeast China, and the curve with noise reduction based on EMD. In the original net load curve, there are more fluctuations within the narrow range generating many extreme points, which make following the curve analysis difficult and affect the analysis efficiency.

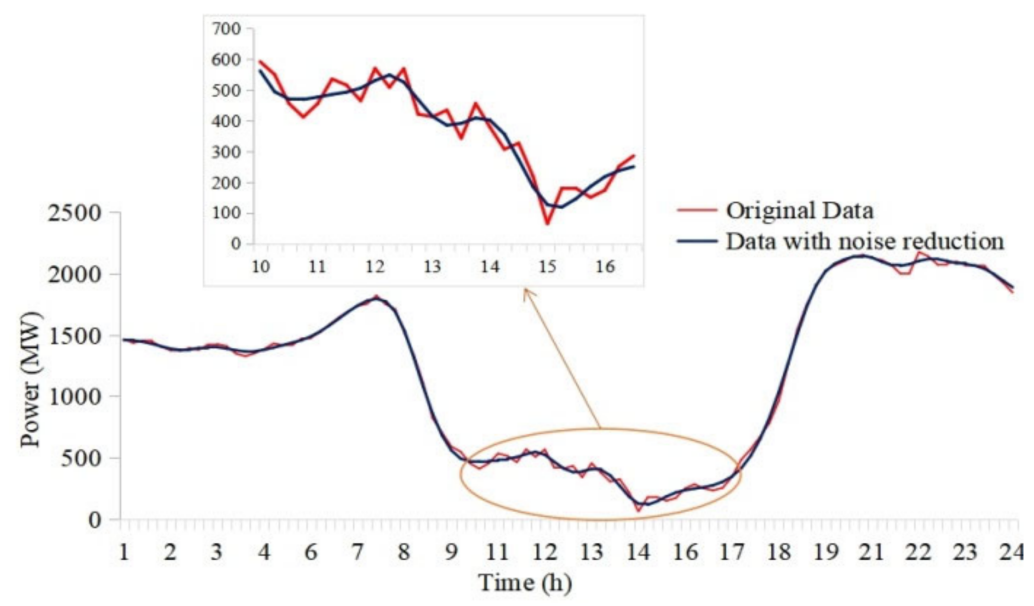

Figure 4. Comparison diagram of the net load with noise reduction and the original curve.

The net load curves decomposed through EMD are shown in Figure 5, in which the plots show high frequency components, medium frequency components, low frequency components, and margin. To reduce the noise of the curve, the high frequency component, the margin, is removed and the remaining components, IMF1 to IMF4, are added as the noise-reduced net load curve, shown in Figure 4 in red. Compared with the original curve 
in dark blue, shown in Figure 4, the noise-reduced net load curve maintains the changing trends of the original, and fluctuations are weakened within a narrow range.
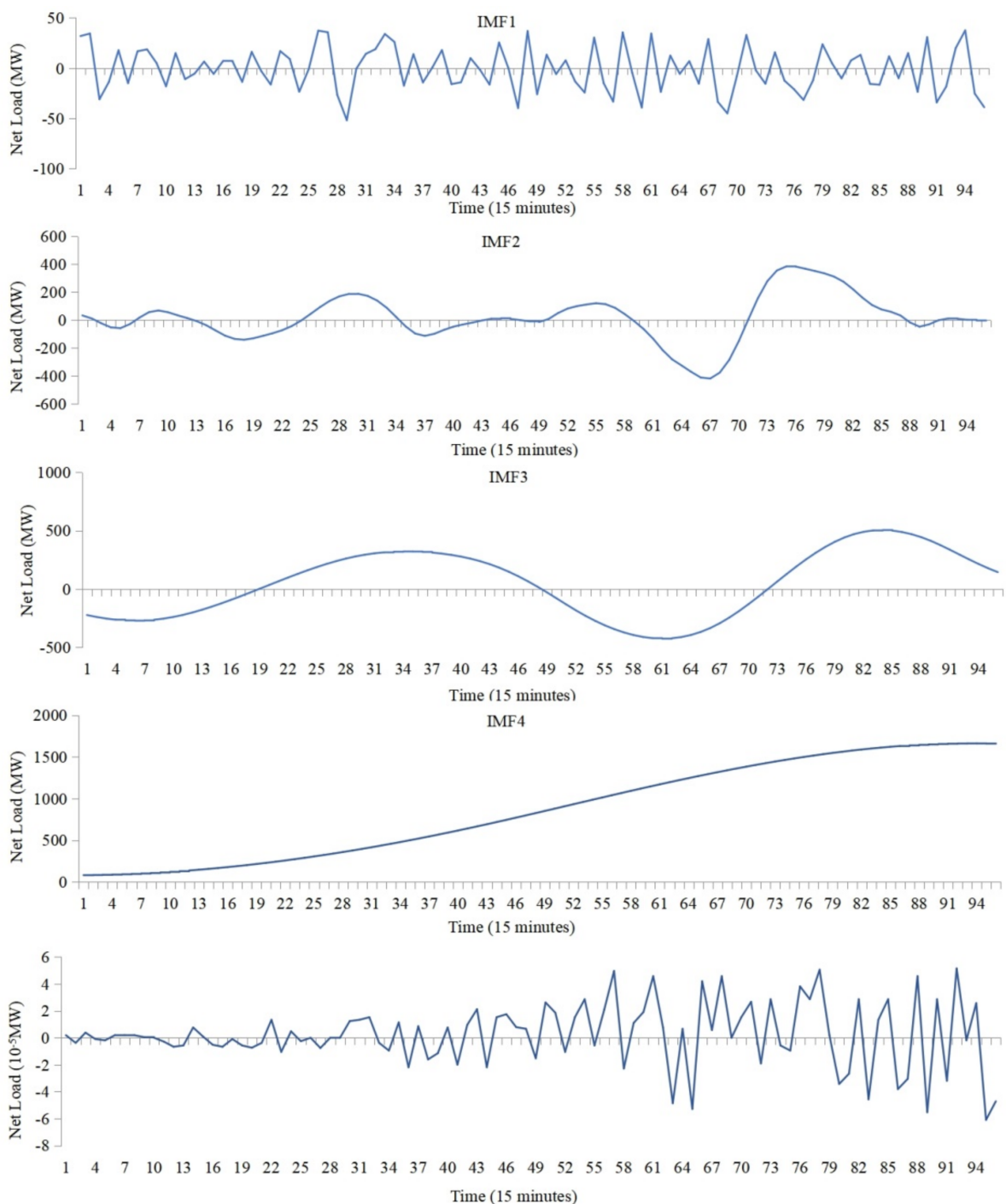

Figure 5. The IMF components and margin of the net load.

\subsection{Numerical Characteristic Calculation}

The slope symbol of the adjacent points can reflect the changing direction of the net loads. In a period, if the net loads have monotonous changing tendency, the slope symbol of the adjacent points is the same. If the slope symbol of the adjacent points is plus or minus, the net loads have a fluctuated changing tendency. Thus, the ratio of slope symbol changing times to the data length can reflect the numerical fluctuation of the net loads. The calculation process is shown in Figure 6.

In this section, $S_{\mathrm{N}}$ is the slope of the adjacent points, where $S_{\mathrm{N}}(l)=n l(l+1)-n l(l)$. If the symbol of $S_{N}$ is plus, the net load presents an increasing trend and reducing trend conversely. The same and different symbol of the adjacent $S_{\mathrm{N}}$ can be presented by 0 and 1 , respectively, and the symbol vector $\mathbf{s y m}^{S_{N}}=\left\{s y m^{S_{N}}\right\}$ is achieved. The ratio of slope symbol changing times to the data length, $R^{S_{N}}$, can be calculated in Equation (8):

$$
R_{l, l+k}^{S_{\mathrm{N}}}=\frac{\sum_{k=1}^{N-l} s y m_{l+k}^{S_{\mathrm{N}}}}{k}
$$


where $l$ is the starting point of the calculated data and $l=1,2, \ldots, N-1 ; k$ is the length of the calculated data and $k=1,2, \ldots, N-l ; N$ is the total number of the net loads.

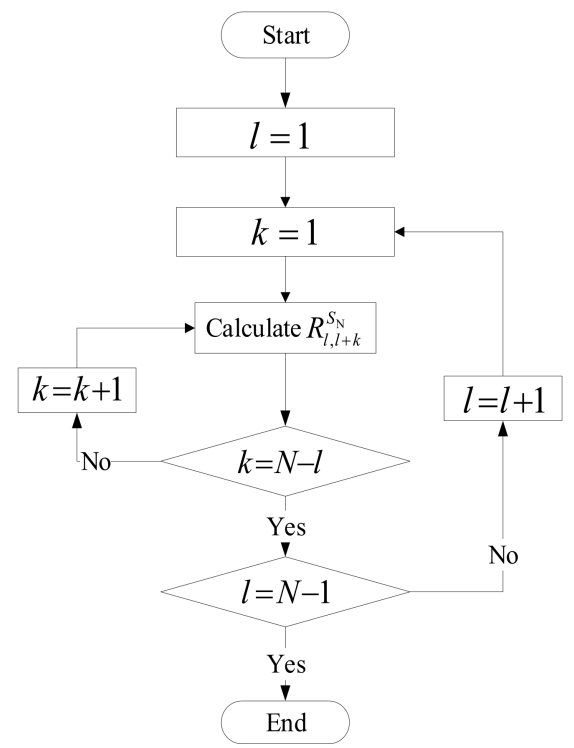

Figure 6. Calculation process of the ratio of slope symbol variation to data length.

\subsection{Net Load Complexity Calculation}

In this paper, the sample entropy (SampEn) proposed by Richman [26,27] is utilized to calculate the complexity of the net load time series. The numerical value of SampEn is in proportion to the net load complexities. The more complex the net loads, the larger the SampEn and vice versa [28]. Based on the SampEn, the complexity calculation of the divided net load subseries is shown in Equation (9):

$$
\left\{\begin{array}{l}
A_{\mathrm{N} i}^{m_{\mathrm{N}}}\left(r_{N}\right)=\frac{\operatorname{count}\left(D_{\mathrm{N}_{i j}}^{m_{\mathrm{N}}}\left(L_{\mathrm{N}}(i), L_{\mathrm{N}}(j)\right)<r_{\mathrm{N}}\right)}{T_{\mathrm{N}}-m_{\mathrm{N}}} \\
A_{\mathrm{Avg}}^{m_{\mathrm{N}}}\left(r_{\mathrm{N}}\right)=\left(\sum_{i=1}^{T_{\mathrm{N}}-m_{\mathrm{N}}+1} A_{\mathrm{N} i}^{m_{\mathrm{N}}}\left(r_{\mathrm{N}}\right)\right) /\left(T_{\mathrm{N}}-m_{\mathrm{N}}+1\right) \\
\delta_{\mathrm{N}}^{r_{\mathrm{N}}, m_{\mathrm{N}}, T_{\mathrm{N}}}=-\ln \left(A_{\mathrm{Avg}}^{m_{\mathrm{N}}+1}\left(r_{\mathrm{N}}\right) / A_{\mathrm{Avg}}^{m_{\mathrm{N}}}\left(r_{\mathrm{N}}\right)\right)
\end{array}\right.
$$

where $\delta_{\mathrm{N}}^{r_{\mathrm{N}}, m_{\mathrm{N}}, T_{\mathrm{N}}}$ is the complexity of the time series; $m_{\mathrm{N}}$ is the dimension of subsequence; $T_{\mathrm{N}}$ is the length of the net load subseries; $i, j \in\left\{1,2, \ldots, T_{\mathrm{N}}-m_{\mathrm{N}}+1\right\}$, in which $i \neq j$; $D_{\mathrm{N}_{i j}}^{m_{\mathrm{N}}}\left(L_{\mathrm{N}}(i), L_{\mathrm{N}}(j)\right)$ is the maximum distance of $L_{\mathrm{N}}(i)$ and $L_{\mathrm{N}}(j)$; and $r_{\mathrm{NL}}$ means the tolerance level and is set at 0.2 times the standard deviation of the time series.

\subsection{Quantification of the Net Load Time-Series Characteristics}

In this paper, we consider both the numerical characteristic and the complexity of the net loads equally important. The mean value is taken as the quantitative index, which is expressed as $\phi_{\tau}$, and $\tau$ is the number of time intervals. The calculation process follows:

(1) Decompose the net load curve based on EMD and extract the noise reduction curve with the high frequency component reduction.

(2) Analyze the numerical characteristic of the net load curve and calculate the ratio of slope symbol changing time to the data length.

(3) Based on the net load time division results, calculate the complexity of the net load subsequence with SampEn.

(4) Synthesize the time division results, numerical characteristic, and complexity; the quantitative index $\phi_{\tau}$ is calculated. 


\section{Time-Interval-Varying Optimal Power Dispatch Model}

\subsection{Time-Interval-Varying Optimal Power Dispatch Strategy Description}

The $\phi$ index reflects the fluctuation and complexity of the net load subseries. The larger $\phi$ is, the more fluctuant and complex it is. Conversely, the smaller $\phi$ is, the more obvious the increase and decrease trend and the less complex it is. The large or small thresholds of $\phi$ are expressed by $\zeta$ and it always is the mean value. However, if there are order of magnitude differences among different $\phi$, the order of magnitude is preferred as the $\zeta$. If $\phi_{\tau} \geq \zeta$ during time $\tau$, the variations of changing direction are more and there is no obvious changing tendency. Thus, in this time interval, the traditional thermal generators ensure continuous and stable operation and the pumped storage and battery energy storage cooperated to assist to achieve power balance. If $\phi_{\tau}<\zeta$, the changing direction variations are less and there is obvious increasing or decreasing tendency of the net loads. Thus, in order to respond to the continue increase or decrease of the net loads during time $\tau$, the power outputs of the traditional thermal generator need to ramp up and down and cooperate with the pumped storage and battery energy storage (BES) to achieve power balance. The thermal operation mode in the proposed time-interval-varying optimal power dispatch strategy is shown in Equations (10)-(12).

$$
\begin{gathered}
\sum_{p=1}^{G} P_{p, t}-P_{\mathrm{ch}, t}^{\mathrm{B}}+P_{\mathrm{dis}, t}^{\mathrm{B}}+\sum_{h=1}^{H} P_{\mathrm{PS}, h, t}^{\mathrm{G}}-\sum_{h=1}^{H} P_{\mathrm{PS}, h, t}^{\mathrm{P}}-L_{\mathrm{N}, t}=0 \\
P_{p, t_{\tau}}=P_{p, t}, t \in\left(t_{\tau}, t_{\tau+1}\right), \phi_{\tau} \geq \zeta \\
P_{p}^{\mathrm{min}} \leq P_{p, t} \leq P_{p}^{\max }, t \in\left(t_{\tau}, t_{\tau+1}\right), \phi_{\tau}<\zeta
\end{gathered}
$$

where $P_{p, t}$ is the power output of the pth thermal generator; $P_{p}^{\min }$ and $P_{p}^{\max }$ are the minimum and maximum power of the pth thermal generator, respectively; $G$ is the number of controllable generators; $H$ is the number of the pumped storage; $P_{\mathrm{PS}, h, t}^{\mathrm{G}}$ is the generating power of the pumped storage; $P_{\mathrm{PS}, h, t}^{\mathrm{P}}$ is the pumping power of the pumped storage; $P_{\mathrm{ch}, t}^{\mathrm{B}}$ and $P_{\text {dis,t }}^{\mathrm{B}}$ are the charging and discharging power of the BES; $\sum_{p=1}^{G} P_{p, t}$ is the power outputs of thermal generators; and $\sum_{h=1}^{H} P_{\mathrm{PS}, h, t}^{\mathrm{G}}$ and $\sum_{h=1}^{H} P_{\mathrm{PS}, h, t}^{\mathrm{P}}$ are the generating power and pumping power of the pumped storage, respectively. $L_{\mathrm{N}, t}$ is the net load at time $t ; P_{\mathrm{L}, t}$ is the traditional load at time $t ; t_{\tau}$ is starting point of the net load subsequence during time interval $\tau ; t_{\tau+1}$ is the ending point of the net load subsequence during time interval $\tau$, that is, the starting point of the net load subsequence during time interval $\tau+1$; and $\Delta t_{\tau}$ is the length of the $\tau$ th net load subsequence. Furthermore, in order to ensure the power output consecutiveness of the thermal generator during each time interval, the power outputs at the starting and ending time points during the adjacent periods should be the same, that is, $P_{p, t_{\tau}}=P_{p, t_{\tau}+\Delta t_{\tau}}$.

\subsection{Time-Interval-Varying Optimal Power Dispatch Model}

\subsubsection{Objective Functions}

The objective functions in the proposed power dispatch strategy are the operation cost of the thermal generators $f_{\mathrm{TH}}$, the operation cost of the pumped storage $f_{\mathrm{PS}}$, the ramping operation cost of the thermal generators $f_{\mathrm{RP}}$, and the environmental cost $f_{\mathrm{EN}}$ caused by the ramping operation of the thermal generators, which include the emissions of sulfur dioxide $\left(\mathrm{SO}_{2}\right)$, oxynitride $\left(\mathrm{NO}_{\mathrm{X}}\right)$, carbon dioxide $\left(\mathrm{CO}_{2}\right)$, and smoke dust. The equations follow:

$$
f_{\mathrm{TH}}=\sum_{t=1}^{T} \sum_{p}^{G}\left(\alpha_{p} \times P_{p, t}^{2}+\beta_{p} \times P_{p, t}+\gamma_{p}\right)
$$




$$
\begin{gathered}
f_{\mathrm{PS}}=\sum_{t=1}^{T} \sum_{h}^{H}\left(c_{\mathrm{PS}}^{\mathrm{P}} \times \mathrm{S}_{\mathrm{PS}}^{\mathrm{P}}+c_{\mathrm{PS}}^{\mathrm{G}} \times \mathrm{S}_{\mathrm{PS}}^{\mathrm{G}}\right)+\sum_{t=1}^{T-1}\left(c_{\mathrm{PS}}^{\mathrm{PG}} \times \mathrm{S}_{\mathrm{PS}, t, t+1}^{\mathrm{PG}}\right) \\
f_{\mathrm{RP}}=\sum_{p=1}^{G}\left(c_{p}^{\mathrm{RU}} \times \sum_{t=1}^{T} U_{p}^{\mathrm{RU}}\right)+\sum_{p=1}^{G}\left(c_{p}^{\mathrm{RD}} \times \sum_{t=1}^{T} D_{p}^{\mathrm{RD}}\right) \\
f_{\mathrm{EN}}=\sigma \sum_{t=1}^{T} \sum_{p}^{G} P_{p, t}
\end{gathered}
$$

where the $T$ is the total number of time intervals; $\alpha_{p}, \beta_{p}$, and $\gamma_{p}$ are the cost coefficients of the thermal generator $p ; c_{\mathrm{PS}}^{\mathrm{P}}, c_{\mathrm{PS}}^{\mathrm{G}}$, and $c_{\mathrm{PS}}^{\mathrm{PG}}$ are the cost coefficients of the pumped storage, which correspond to the pumping state $S_{\mathrm{PS}}^{\mathrm{P}}$, generating state $\mathrm{S}_{\mathrm{PS}}^{\mathrm{G}}$, and status switching $\mathrm{S}_{\mathrm{PS}, t, t+1}^{\mathrm{PG}}$, respectively; $c_{p}^{\mathrm{RU}}$ and $c_{p}^{\mathrm{RD}}$ are the ramping up and down cost coefficients of thermal generators; $\boldsymbol{\sigma}$ is the environmental cost coefficient vector; and $U_{p}^{\mathrm{RU}}=\max \left(P_{p, t}-P_{p, t-1}, 0\right)$ and $D_{p}^{\mathrm{RD}}=\max \left(P_{p, t-1}-P_{p, t}, 0\right)$ are the ramping up and down power outputs of the thermal generator, respectively.

\subsubsection{Constraints of the Dispatch Model}

Power Balance Constraints

The power balance constraints are shown in Equations (10) and (17):

$$
L_{\mathrm{N}, t}=P_{\mathrm{L}, t}-\left(\sum_{w=1}^{W} P_{\mathrm{W}, w, t}^{\mathrm{pre}}+\sum_{s=1}^{S} P_{\mathrm{S}, s, t}^{\mathrm{pre}}\right)
$$

where the $P_{\mathrm{W}, w, t}^{\mathrm{pre}}$ is the predicted wind power; $W$ is the total number of wind farms; $P_{\mathrm{S}, s, t}^{\mathrm{pre}}$ is the predicted photovoltaic power; and $S$ is the total number of photovoltaic power plants.

\section{Constraints of Thermal Power Outputs}

The thermal power output constraints are shown in Equation (18):

$$
\left\{\begin{array}{l}
\sum_{p=1}^{G} P_{p, t}-P_{\mathrm{ch}, t}^{B}+P_{\mathrm{dis}, t}^{B}+\sum_{h=1}^{H} P_{\mathrm{PS}, h, t}^{G}-\sum_{h=1}^{H} P_{\mathrm{PS}, h, t}^{P}-L_{N, t}=0 \\
\phi_{\tau} \geq \zeta: P_{p, t_{\tau}}=P_{p, t} \\
\phi_{\tau}<\zeta: P_{p}^{\min } \leq P_{p, t} \leq P_{p}^{\max } \\
P_{p, t_{\tau}}=P_{p, t_{\tau}+\Delta t_{\tau}} \\
P_{p, t}-P_{p, t-1} \leq R U_{p} \\
P_{p, t-1}-P_{p, t} \leq R D_{p} \\
\operatorname{Pr}\left\{\sum_{p=1}^{G} P_{p, t}^{\mathrm{UR}} \geq \sum_{w=1}^{W}\left(P_{\mathrm{W}, w, t}^{\mathrm{pre}}-P_{\mathrm{W}, w, t}\right)+\sum_{s=1}^{S} P_{\mathrm{S}, s, t}^{\mathrm{UR}}\right\} \geq \rho \\
\operatorname{Pr}\left\{\sum_{p=1}^{G} P_{p, t}^{\mathrm{DR}}, \geq, \sum_{w=1}^{W},\left(P_{\mathrm{W}, w, t}-P_{\mathrm{W}, w, t}^{\mathrm{pre}}\right)+\sum_{s i=1}^{S} P_{\mathrm{S}, s, t}^{\mathrm{DR}}\right\} \geq \rho \\
P_{p, t}^{\mathrm{UR}} \leq \min \left(P_{p}^{\max }-P_{p, t}, R U_{p}\right) \\
P_{p, t}^{\mathrm{DR}} \leq \min \left(P_{p, t}-P_{p}^{\min }, R D_{p}\right)
\end{array}\right.
$$

where $t \in\left(t_{\tau}, t_{\tau+1}\right), R U_{p}$, and $R D_{p}$ are the upper and lower limit of the thermal ramp power, respectively; $\rho$ is the confidence; $P_{\mathrm{W}, w, t}$ is the actual wind power output; $P_{p, t}^{\mathrm{UR}}$ and $P_{p, t}^{\mathrm{DR}}$ are the up and down reserve power of the thermal generator; and $P_{\mathrm{S}, s, t}^{\mathrm{UR}}$ and $P_{\mathrm{S}, s, t}^{\mathrm{DR}}$ are the up and down reserve power of the photovoltaic. Based on [29], the time scale of photovoltaic fluctuation is always $5 \mathrm{~min}$; thus, in the proposed day-ahead power dispatch strategy, the up and down reserve power of the photovoltaic is $\pm 5 \%$ of the predicted power. 
The probabilistic model with uncertain variables in Equation (18) is solved by the chance constraint and fractile in probability theory [30].

Constraints of the Pumped Storage

The constraints of pumped storage are shown in Equation (19):

$$
\left\{\begin{array}{l}
P_{\mathrm{PS}, h}^{\mathrm{G}, \mathrm{min}} \leq P_{\mathrm{PS}, h, t}^{\mathrm{G}} \leq P_{\mathrm{PS}, h}^{\mathrm{G}, \max } \\
P_{\mathrm{PS}, h}^{\mathrm{P}, \mathrm{min}} \leq P_{\mathrm{PS}, h, t}^{\mathrm{P}} \leq P_{\mathrm{PS}, h}^{\mathrm{P}, h} \\
H_{h, t+1}=H_{h, t}+\Delta t \times\left(P_{\mathrm{PS}, h, t}^{\mathrm{P}} \times \gamma_{\mathrm{P}}-P_{\mathrm{PS}, h, t}^{\mathrm{G}} / \gamma_{\mathrm{G}}\right) \\
H_{h}^{\min } \leq H_{h, t} \leq H_{h}^{\max } \\
S\left(P_{\mathrm{PS}, h, t}^{\mathrm{P}}\right) \cdot S\left(P_{\mathrm{PS}, h, t}^{\mathrm{G}}\right)=0
\end{array}\right.
$$

where $P_{\mathrm{PS}, h}^{\mathrm{G}, \mathrm{min}}$ and $P_{\mathrm{PS}, h}^{\mathrm{G}, \mathrm{max}}$ are the upper and lower limit of pumped storage generating power, respectively; $P_{\mathrm{PS}, h}^{\mathrm{P}, \min }$ and $P_{\mathrm{PS}, h}^{\mathrm{P}, \mathrm{max}}$ are the upper and lower limit of the pumped storage pumping power, respectively; $H_{h, t}$ is the capacity of the reservoir at time $t ; H_{h}^{\max }$ and $H_{h}^{\min }$ are the upper and lower limit of the capacity, respectively; and $\gamma_{\mathrm{P}}$ and $\gamma_{\mathrm{G}}$ are the generating and pumping efficiency of the pumped storage.

\section{Constraints of BES}

The constraints of BES are shown in Equation (20):

$$
\left\{\begin{array}{l}
S O C^{\mathrm{B}, \min } \leq S O C_{t}^{\mathrm{B}} \leq S O C^{\mathrm{B}, \max } \\
E_{\mathrm{Cap}, 1}^{\mathrm{B}}=E_{\mathrm{Cap}, T}^{\mathrm{B}} \\
P_{\mathrm{ch}, t}^{\mathrm{B}} \cdot P_{\mathrm{dis}, t}^{\mathrm{B}}=0 \\
E_{\mathrm{Cap}, t}^{\mathrm{B}}=E_{\mathrm{Cap}, t-1}^{\mathrm{B}}-P_{\mathrm{dis}, t}^{\mathrm{B}} / \eta_{\mathrm{dis}}^{\mathrm{B}} \times \Delta t \\
E_{\mathrm{Cap}, t}^{\mathrm{B}}=E_{\text {Cap }, t-1}^{\mathrm{B}}+P_{\mathrm{ch}, t}^{\mathrm{B}} \times \eta_{\mathrm{ch}}^{\mathrm{B}} \times \Delta t \\
S O C_{t}^{\mathrm{B}}=E_{\text {Cap }, t}^{\mathrm{B}} / E_{\text {Cap }}^{\mathrm{B}, \mathrm{R}}
\end{array}\right.
$$

where $S O C_{t}^{\mathrm{B}, \min }$ and $S O C_{t}^{\mathrm{B}, \max }$ are the minimum and maximum state of charge, respectively; $S O C_{t}^{\mathrm{B}}$ is the state of charge at time $t ; E_{\mathrm{Cap}, 1}^{\mathrm{B}}$ and $E_{\mathrm{Cap}, T}^{\mathrm{B}}$ are the capacity at the starting and ending time interval, respectively; $P_{c h, t}^{\mathrm{B}}$ and $P_{d i s, t}^{\mathrm{B}}$ are the charging and discharging power of $\mathrm{BES}$ at time $t$, respectively; $E_{\mathrm{Cap}}^{\mathrm{B}, \mathrm{R}}$ is the related capacity of the $\mathrm{BES} ; \eta_{\mathrm{dis}}^{\mathrm{B}}$ and $\eta_{\mathrm{ch}}^{\mathrm{B}}$ are the discharging and charging efficiency of BES; and $\Delta t$ is the time interval.

\subsection{The Solving Algorithm and Process}

The solving algorithm and process of the proposed time-interval-varying optimal power dispatch model are shown in Figure 7.

Firstly, the conventional loads, wind power, and photovoltaic are predicted and the net loads are achieved. Secondly, based on the RMT, the net loads are divided according to their characteristics and the time-series characteristics are quantified through EMD and SampEn. Thirdly, based on the quantified indexes, the operation modes of thermal generators and energy storages are determined and the time-interval-varying optimal power dispatch model is built. Finally, the primal-dual interior point method [31] is chosen to solve the proposed dispatch model. 


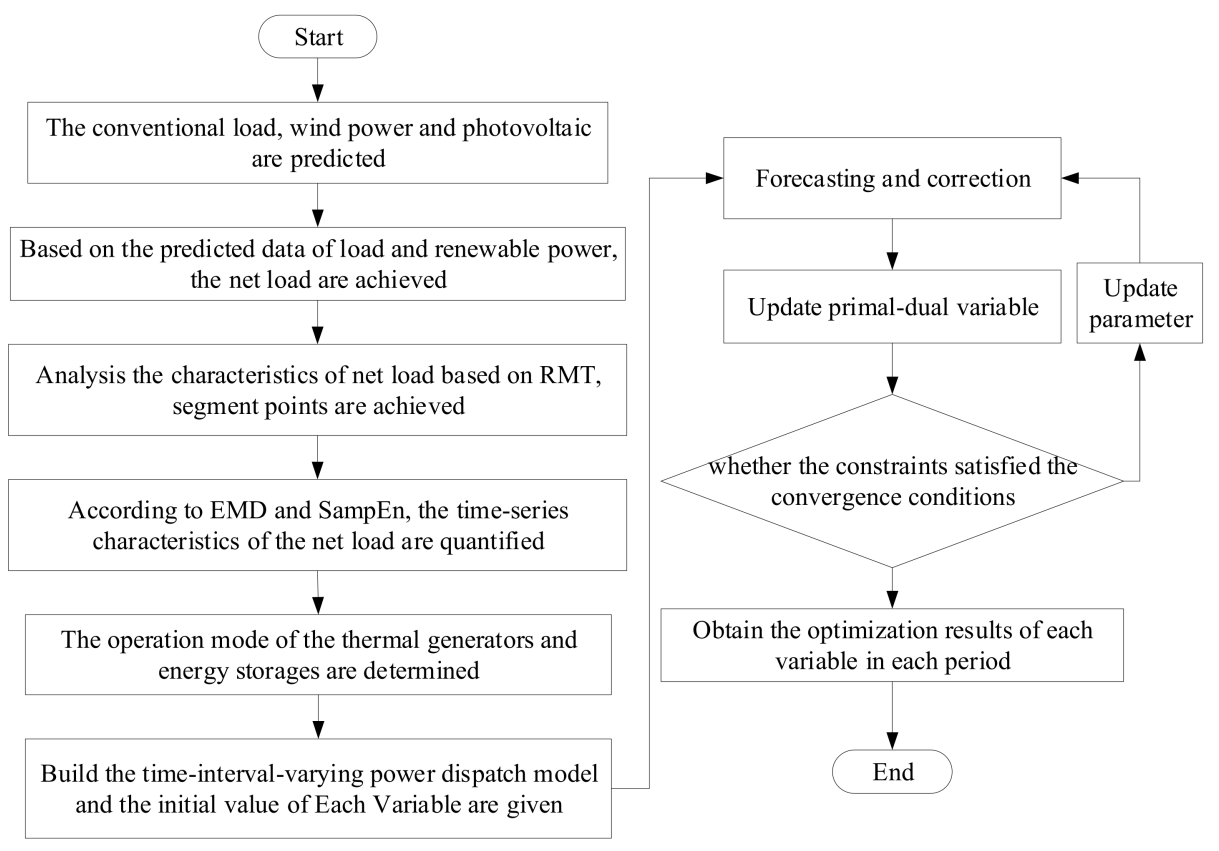

Figure 7. Algorithm and flow chart for solving the time-interval-varying power dispatch model.

\section{Case Study}

\subsection{Data of the Provincial Power Grid}

An actual provincial power grid in 2019 in northeast China is developed to carry out the case study. There are 103 thermal plants and the maximum capacity of schedulable thermal power is $26,800 \mathrm{MW}$. There are 103 wind power plants and the maximum schedulable capacity is $8594 \mathrm{MW}$. There is one pumped storage with the maximum schedulable capacity of $2000 \mathrm{MW}$. There are 90 photovoltaic plants with the maximum schedulable capacity of $3030 \mathrm{MW}$. The number of hydraulic power plants is 15 and the total installed capacity is 620.5 MW. There is one nuclear power plant with the installed capacity of 2237.58 MW. The maximum daily load in 2019 is 34,000 MW; thus, the penetration of renewable energy is $34.19 \%$. The power plants are tallied according to their locations and sizes of capacity; thus, the capacity data of the thermal power plants are shown in Table 1 and the capacity data of wind power plants are shown in Table 2.

Table 1. Thermal power installed capacity of the provincial power grid.

\begin{tabular}{ccc}
\hline Power Plant & Number & Maximum Capacity (MW) \\
\hline A & 8 & 2600 \\
B & 2 & 1200 \\
C & 4 & 1400 \\
D & 14 & 1970 \\
E & 2 & 600 \\
F & 3 & 1400 \\
G & 6 & 2400 \\
H & 2 & 1200 \\
I & 8 & 2340 \\
J & 8 & 1700 \\
K & 10 & 2500 \\
L & 12 & 1000 \\
M & 4 & 1300 \\
N & 6 & 790 \\
\hline
\end{tabular}


Table 1. Cont.

\begin{tabular}{ccc}
\hline Power Plant & Number & Maximum Capacity (MW) \\
\hline O & 2 & 600 \\
P & 4 & 500 \\
Q & 4 & 1900 \\
R & 2 & 700 \\
S & 2 & 700 \\
Total & 103 & 26,800 \\
\hline
\end{tabular}

Table 2. Wind power installed capacity of the provincial power grid.

\begin{tabular}{ccc}
\hline Power Plant & Number & Maximum Capacity (MW) \\
\hline W1 & 1238 & 1238 \\
W2 & 1915 & 1915 \\
W3 & 1504 & 1504 \\
W4 & 2336 & 2336 \\
W5 & 1601 & 1601 \\
\hline
\end{tabular}

The operation costs of wind power, photovoltaic, nuclear power, and pumped storage are ignored. The nuclear power plant operates at a constant power supply status. The cost coefficients of the reserve power and the ramping power are both $100 \mathrm{CNY} / \mathrm{MW}$. The capacity of battery energy storage in this province is $1000 \mathrm{MW}$. The net load curve of the provincial power system is shown in Figure 8.

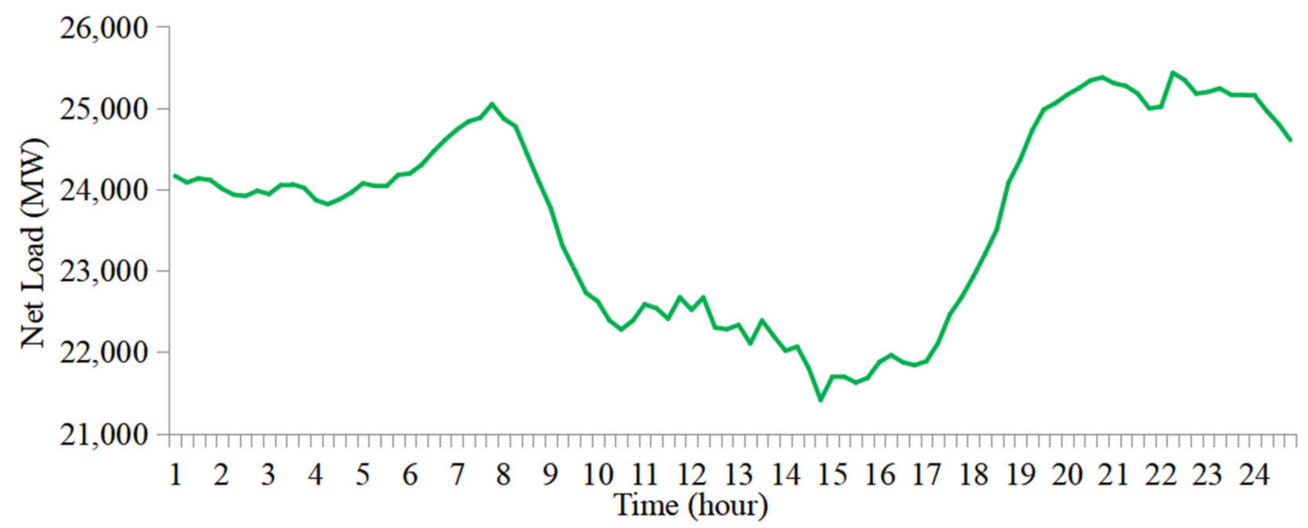

Figure 8. Net load curve of the provincial power system.

\subsection{Time-Series Characteristic of the Provincial Net Load}

\subsubsection{Time Division Based RMT}

The actual net load on 20 April 2019 in the provincial power gird is analyzed. The net load curve, the MSR analysis based on RMT, and the time division results are shown in Figure 9, where the net loads are divided in five time intervals. When the system is at a steady state, all the eigenvalues are distributed in the loop and the MSR is a stable value determined by the number of matrix rows and columns. In this paper, the stable value of MSR is 0.86 and the stability range is $0.86 \pm 5 \%$. 


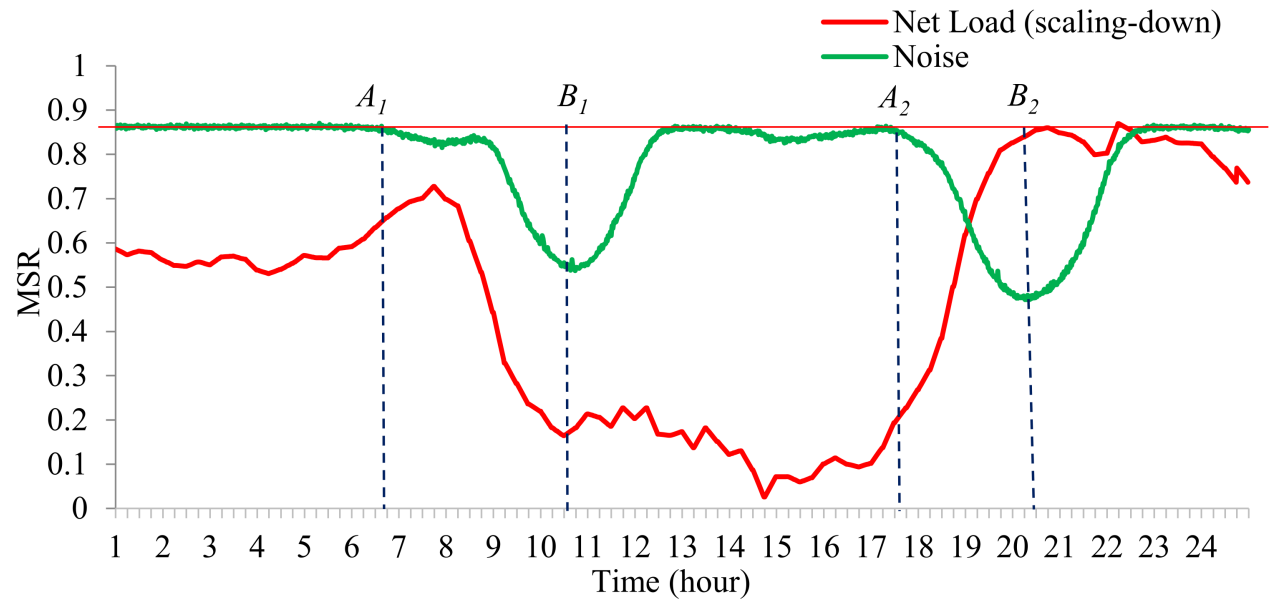

Figure 9. MSR analysis and time division for 20 April.

\subsubsection{Characteristic Analysis of the Each Subseries}

Based on EMD, we can obtain the net load curve with the noise reduction and its comparison diagram with the original curve, which is shown in Figure 4. After the noise reduction by EMD, the numerical and complexity calculation of the net load time series is shown in Table 3.

Table 3. Time-series characteristic analysis of the net load for 20 April.

\begin{tabular}{cccccccc}
\hline Time Interval & Starting Time & Ending Time & $\boldsymbol{R}^{\boldsymbol{S}_{\mathbf{N}}}$ & Proportion of $\boldsymbol{R}^{\boldsymbol{S}_{\mathbf{N}}}$ & SampEn & Proportion of SampEn & $\boldsymbol{\phi}$ \\
\hline 1 & 1 & 6 & 0.14 & $25 \%$ & 0.18 & $27 \%$ & 0.26 \\
2 & 7 & 10 & 0.07 & $13 \%$ & 0.07 & 0.115 \\
3 & 11 & 17 & 0.15 & $27 \%$ & 0.30 & $45 \%$ & $3 \%$ \\
4 & 18 & 20 & 0.00 & 0 & 0.02 & 0.36 \\
5 & 21 & 24 & 0.19 & $35 \%$ & 0.10 & $15 \%$ & 0.25 \\
\hline
\end{tabular}

According to Figure 8, the changing direction of data in the first, third, and fifth time intervals have frequent variations and the data in the second and fourth time intervals have obvious changing tendency, which is in accordance with the proportion of $R^{S_{\mathrm{N}}}$ and the SampEn proportion in Table 3. According to the $R^{S_{N}}$ and SampEn, the time-series characteristic quantification index $\phi$ can be achieved and the mean value of $\phi$ is utilized as the threshold.

\subsection{Time-Interval-Varying Dispatch Results and Benefits Analysis}

The case study is conducted for two cases:

Case 1: The provincial power dispatch under the existing conventional dispatch mode; Case 2: The provincial power dispatch under the proposed time-interval-varying net load power dispatch mode.

\subsubsection{Dispatch Result Analysis of the Two Cases}

With the same starting points and operation accuracy, the solution time for Case 1 and Case 2 is $3467 \mathrm{~s} \mathrm{(57} \mathrm{min}$ and $47 \mathrm{~s}$ ) and $4760 \mathrm{~s} \mathrm{(1} \mathrm{h} 19 \mathrm{~min}$ and $20 \mathrm{~s}$ ), respectively. There are equality constraints of thermal generator operation; thus, the solution time for Case 2 is longer than that in Case 1. The power output curves of thermal generators in Case 1 and Case 2 are shown in Figures 10 and 11. 


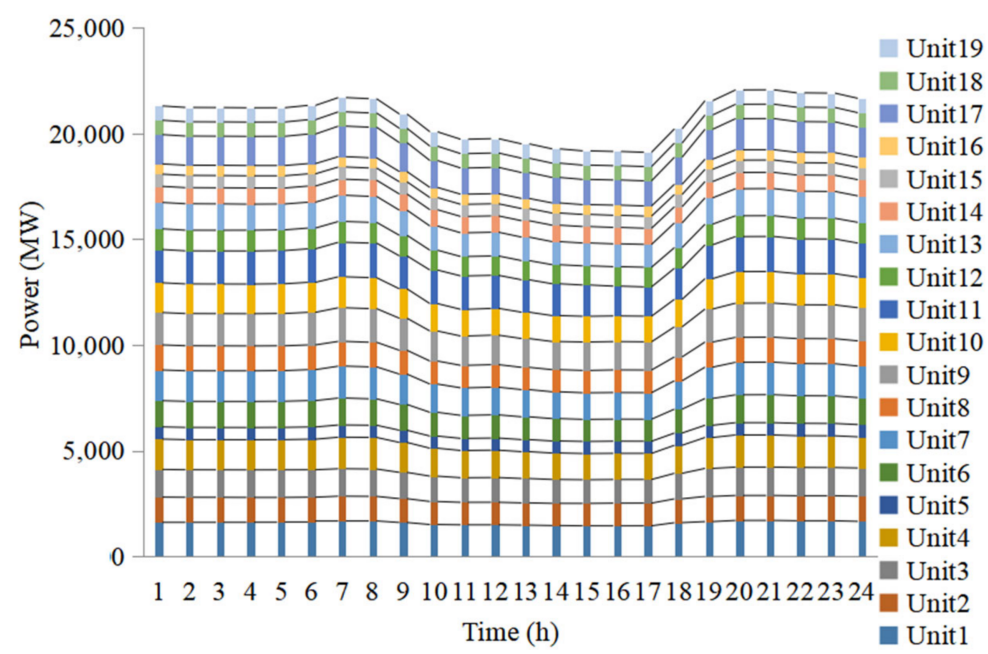

Figure 10. The power outputs of thermal generators in Case 1.

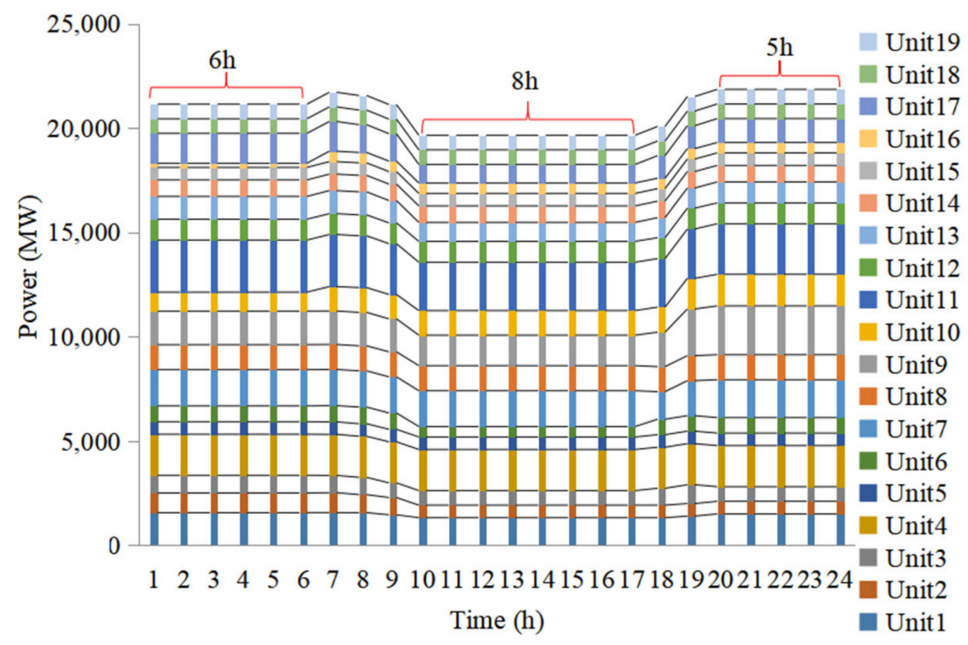

Figure 11. The power outputs of thermal generators in Case 2.

The volatility of each unit can be analyzed by a box plot [32], shown in Figure 12. The length of the box is positive correlation to the volatility of the data. The volatilities of the units in Case 1 are more than that in Case 2. The power outputs of pumped storage and BES in Case 1 and Case 2 are shown in Figures 13 and 14. Their throughput comparisons are shown in Figure 15.

(a)

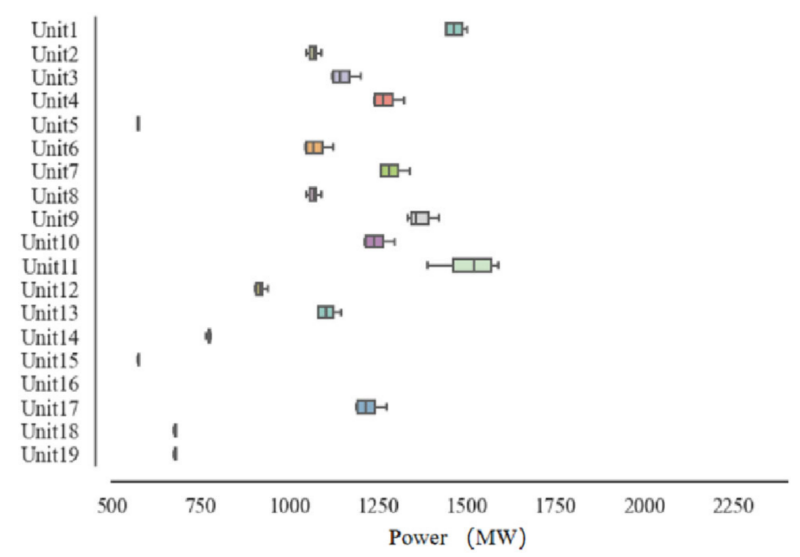

Figure 12. Cont. 
(b)

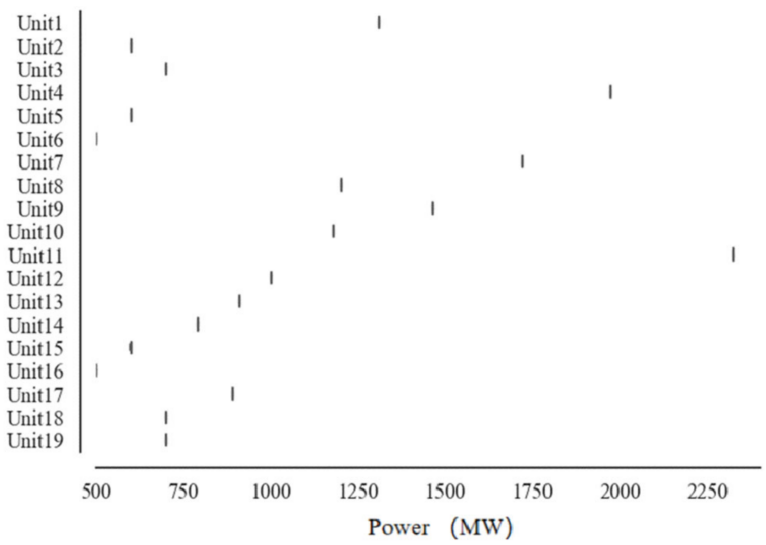

Figure 12. The volatility of the two cases: (a) is Case 1 and (b) is Case 2.

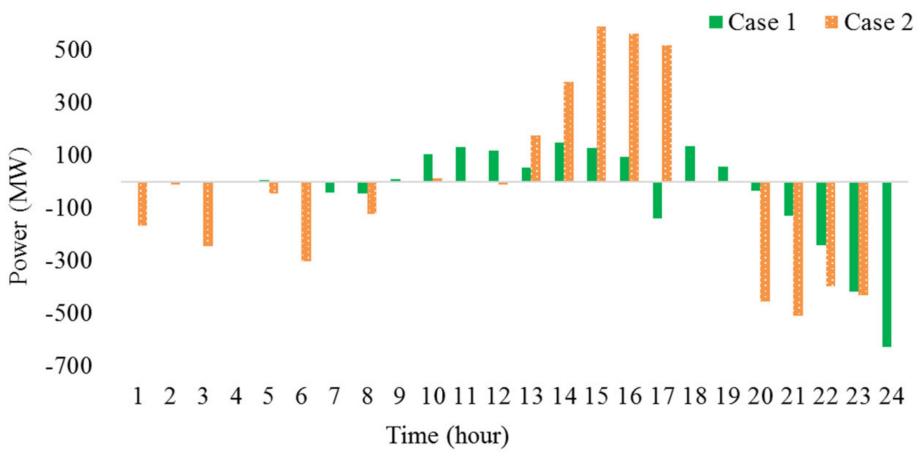

Figure 13. The power outputs of pumped storage in Case 1 and Case 2.

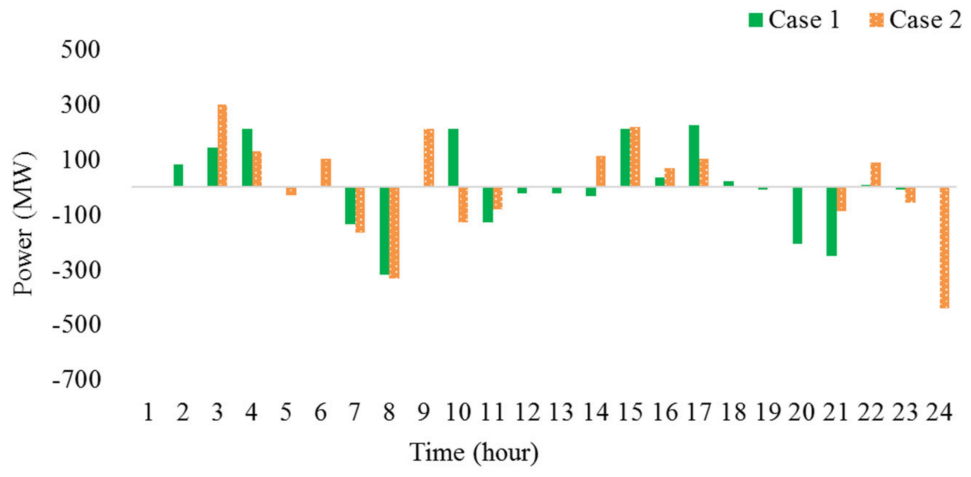

Figure 14. The power outputs of BES in Case 1 and Case 2.

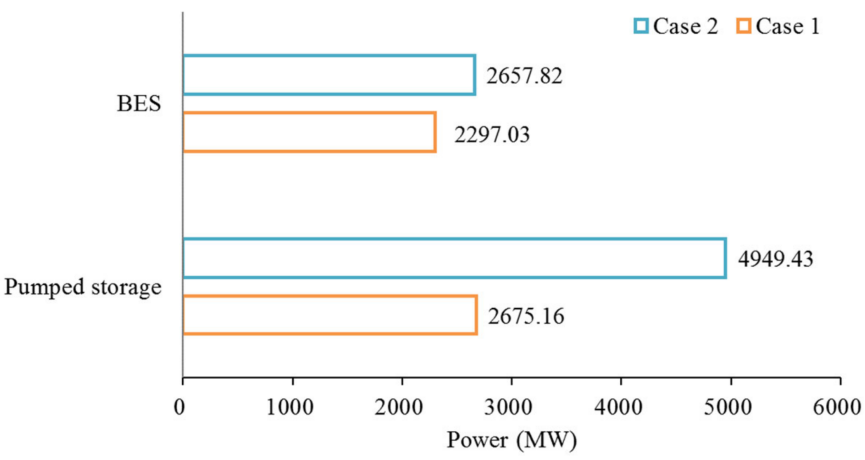

Figure 15. The throughput of pumped storage and BES in Case 1 and Case 2. 
The ramp-up and ramp-down power and their cost for Case 1 and Case 2 are shown in Figures 16 and 17, respectively.

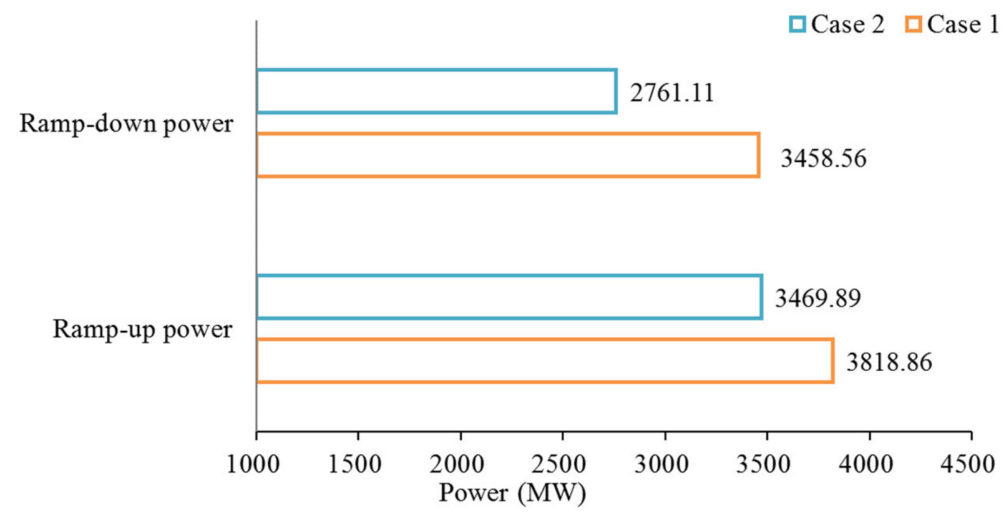

Figure 16. The ramp-up and ramp-down power of Case 1 and Case 2.

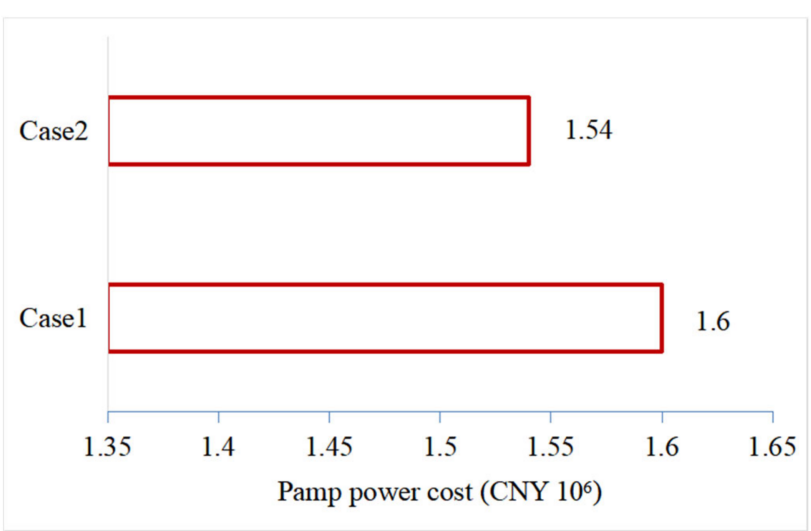

Figure 17. The ramp power cost of Case 1 and Case 2.

A comparison for Case 1 and Case 2 results is shown in Table 4 and the changing percentages are given. Under the time-interval-varying power dispatch mode, the ramping operation of the traditional thermal generators is reduced.

Table 4. Comparison of Case 1 and Case 2 results.

\begin{tabular}{cccc}
\hline & Case 1 & Case 2 & Decreasing Percentage in Case 2 \\
\hline Total operation cost (CNY 10 $\left.{ }^{7}\right)$ & 11.64 & 11.55 & $0.77 \%$ \\
Thermal operation cost (CNY 107) & 11.48 & 11.39 & $0.78 \%$ \\
Ramping power cost (CNY 10 $\left.{ }^{7}\right)$ & 1.60 & 1.54 & $3.75 \%$ \\
Ramp-up power (MW) & 3818.86 & 3469.89 & $9.14 \%$ \\
Ramp-down power (MW) & 3458.56 & 2761.11 & $20.17 \%$ \\
Throughput of pumped storage (MW) & 2675.16 & 4949.43 & $-85.01 \%$ \\
Throughput of BES (MW) & 2297.03 & 2657.82 & $-15.71 \%$ \\
\hline
\end{tabular}

\subsubsection{Environmental Benefit Analysis}

The environmental benefit is analyzed in this section and the environmental cost coefficients are from [14]. The total environmental costs caused by the ramping operation of the thermal generators are analyzed. The results are shown in Figure 18 and the unit is $\mathrm{kg}$. 

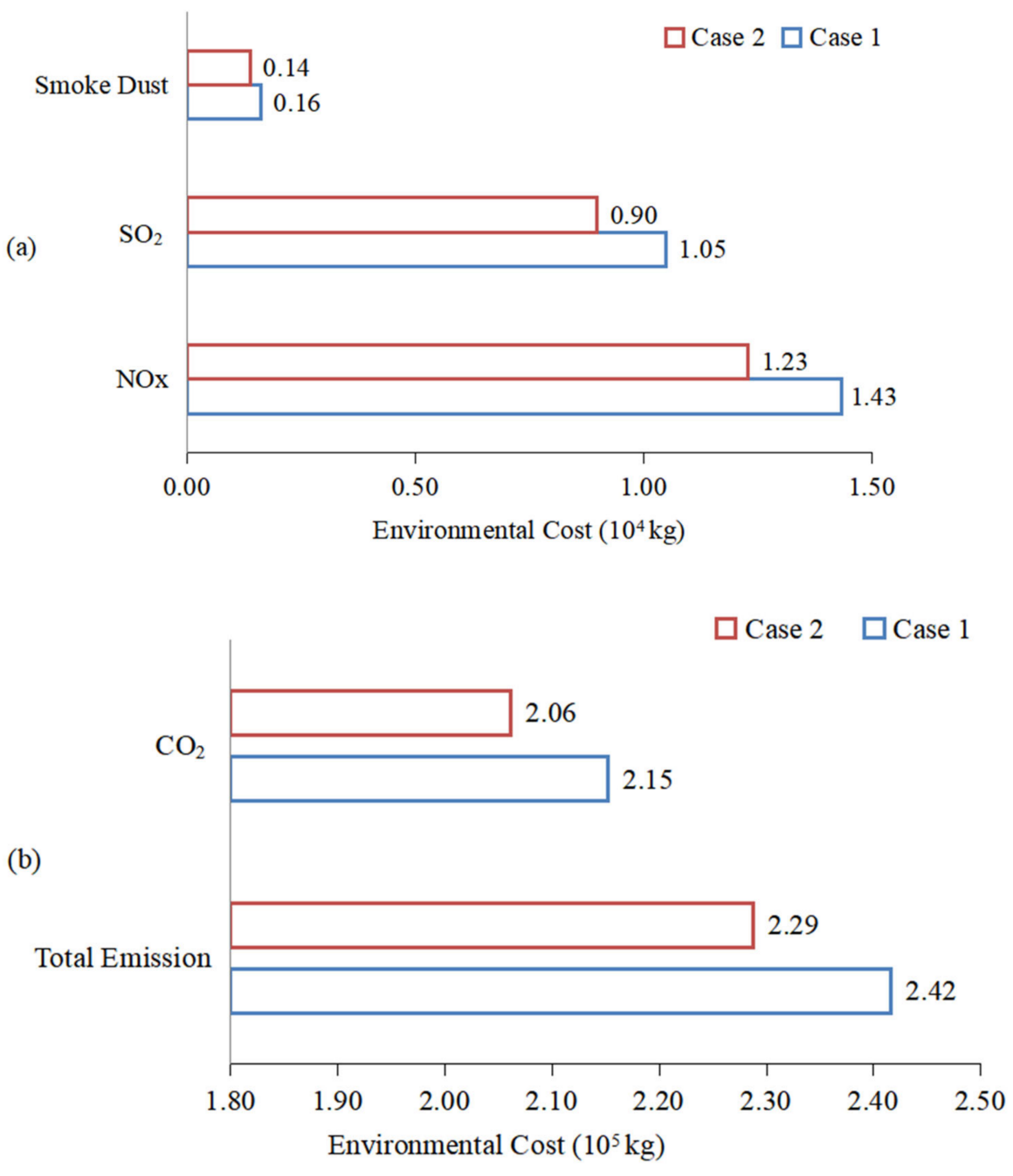

Figure 18. The emissions of $\mathrm{SO}_{2}, \mathrm{NOx}$, smoke dust, $\mathrm{CO}_{2}$, and total environmental costs for each case: (a) is the environment costs of smoke dust, $\mathrm{SO}_{2}$ and $\mathrm{NOx}$; (b) is the environment costs of $\mathrm{CO}_{2}$ and total emission.

\subsection{Case Study Based on the IEEE 39 System}

Discussion Based on the Provincial Power System

To further illustrate the effectiveness of the proposed method in this paper, an improved IEEE 39 system [33] utilized to conduct a 96-time-interval power dispatch optimization (a time period duration is $15 \mathrm{~min}$ ). There is a $700 \mathrm{MW}$ pumped storage, a wind farm with $1000 \mathrm{MW}$ capacity, a $600 \mathrm{MW}$ photovoltaic plant, and a $500 \mathrm{MW}$ battery energy storage. The net load of this system is shown in Figure 19. The case study on the IEEE 39 system is conducted for two cases as follows:

Case 3: Power dispatch under the existing conventional dispatch mode;

Case 4: Power dispatch under the proposed time-interval-varying net load power dispatch mode.

The power outputs of the thermal generators in Case 3 and Case 4 are shown in Figures 20 and 21, respectively. The result comparison is shown in Table 5. 


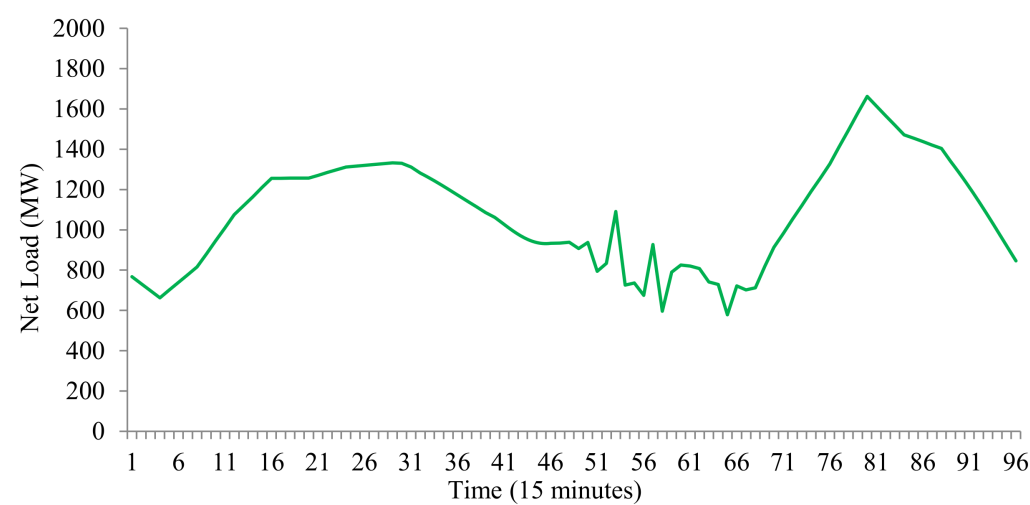

Figure 19. The net load curve of the IEEE 39 system.

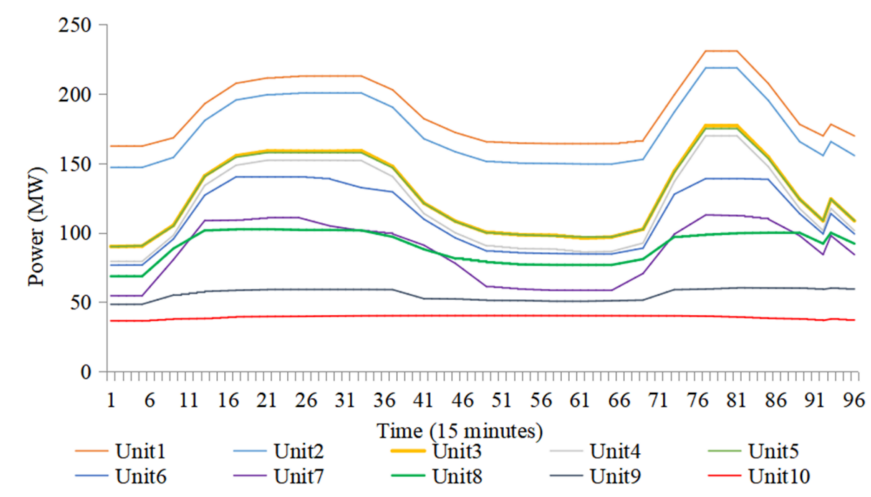

Figure 20. The power outputs of thermal generators in Case 3.

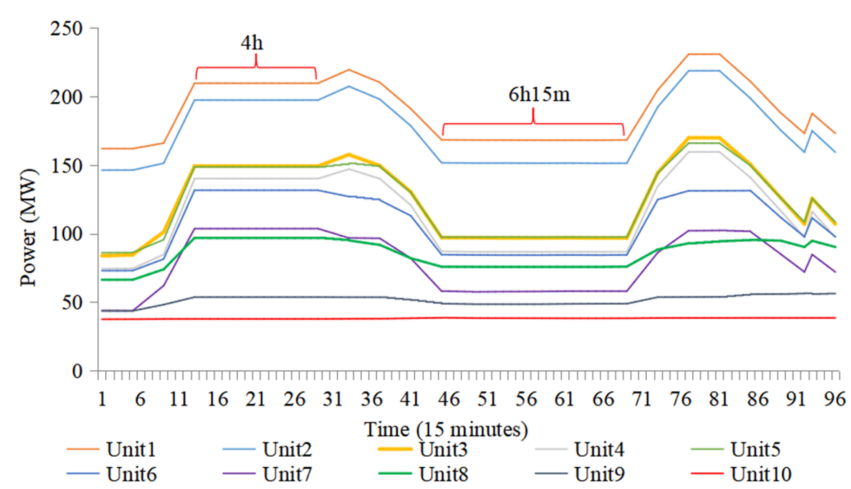

Figure 21. The power outputs of thermal generators in Case 4.

Table 5. Comparison of Case 3 and Case 4 results.

\begin{tabular}{cccc}
\hline & Case 3 & Case 4 & Decreasing Percentage in Case 3 \\
\hline Total operation cost (CNY 10 $\left.{ }^{5}\right)$ & 7.3702 & 7.3572 & $0.18 \%$ \\
Thermal operation cost (CNY 10 $)$ & 7.2060 & 7.2075 & $-0.02 \%$ \\
Ramping power cost (CNY) & $11,920.06$ & $10,225.84$ & $16.57 \%$ \\
Ramp-up power (MW) & 1046.61 & 956.84 & $9.38 \%$ \\
Ramp-down power (MW) & 876.13 & 811.95 & $-3.9 \%$ \\
Throughput of pumped storage (MW) & 938.94 & 1473.88 & $-20.27 \%$ \\
Throughput of BES (MW) & 1381.42 & 1732.56 & \\
\hline
\end{tabular}




\subsection{Discussion}

\subsubsection{Discussion Based on the Provincial Power System}

As Figures 10 and 11 show, in Case 1, Units 14-16 and 19 are the generators with small capacity and poor peak load regulation capability, thus the power output adjustments are less. The outputs of the other generators changed frequently according to net loads. Under the conventional dispatch time division, the thermal power outputs need to be adjusted each hour. However, in Case 2 under the provided dispatch strategy, the dispatch time intervals are divided based on the time-series characteristics of the net loads and for the provincial power grid described above, the conventional day-ahead power dispatch mode is updated to "6-3-8-2-5" power dispatch mode. It can be seen in Figure 11, during the 1-6, 10-17, and 20-24 time intervals, the thermal generators are operating at a constant operation mode. During the 7-9 and 18-19 time intervals, the thermal generators are operating at a net-load-response operation mode, in which the outputs of thermal generators change hourly with the value of net load. The power output adjusting time is totally $5 \mathrm{~h}$. The continuous and stable thermal generating times are 6,8 , and $5 \mathrm{~h}$ in the first, third, and fifth time interval, respectively. As Figure 15 shows, the volatility of thermal generation in Case 2 is better than that in Case 1, obviously.

As Figures 13 and 14 and Table 4 show, in the optimal power dispatch, the pumped storage, and BES are used to cooperate with each other to respond to the net loads. In Case 2, the thermal generators have $19 \mathrm{~h}$ of constant power operation, thus the throughput of pumped storage and BES in Case 2 are more than those in Case 1. The throughput of pumped storage and BES in Case 2 increased $85.01 \%$ and $15.71 \%$, respectively. In Case 2, owing to the "6-3-8-2-5" operation mode, the ramping powers of the thermal generators are reduced. Thus, the ramp-up and ramp-down power in Case 2 are $9.14 \%$ and $20.17 \%$ less than those in Case 1, which, moreover, results in a 3.75\% ramping operation cost decrease in Case 2. The participation and utilization efficiency of pumped storage and BES are increased, which improve the interaction between the energy storages and renewable power energy. From the perspective of economics, the proposed power dispatch strategy in this paper optimized the thermal generating behavior according to the time-series characteristics of net loads. The operation modes of thermal generators with different coal consumption coefficients are determined and rationalized. Thus, the thermal operation cost in Case 2 has 0.78\% reduction and the total operation cost in Case 2 is $0.77 \%$ less than that in Case 1.

As Figure 18 shows, all the noted emissions provided in Case 2 are less than those in Case 1; that is, the environmental benefit with time-interval-varying power dispatch mode is better than the conventional dispatch mode. According to the carbon emission requirements concept of carbon credit, in Case 2, there are 9.06 tons of carbon dioxide less than that in Case 1, and 9.06 carbon credits more than Case 1, which demonstrates that time-interval-varying dispatch mode is in line with international energy development demands.

\subsubsection{Discussion Based on the IEEE 39 System}

As Figure 20 shows, the power outputs of thermal generators in Case 3 need to adjust every $15 \mathrm{~min}$ to respond to the net load demand. In Case 4, under the provided dispatch strategy, the dispatch time intervals are divided based on the time-series characteristics of the net loads and for the IEEE 39 system; there are $4 \mathrm{~h}$ and $6 \mathrm{~h} 15 \mathrm{~min}$ under the constant generation state.

The results of the two cases are shown in Table 5. As there are two time intervals in which the thermal generators are at constant generation state in Case 4, the ramp-up and ramp-down power of thermal generators are reduced $9.38 \%$ and $7.9 \%$ compared to those in Case 3. Thus, the ramping cost in Case 4 is $16.57 \%$ lower than that in Case 3 . In order to ensure power balance of the system, the power output of pumped storage and BES in Case 4 needs to adjust more frequently than that in Case 3 . In Case 4 , the thermal generators have two time intervals operating at constant power output. Thus, the throughput of pumped 
storage and BES are 36.29\% and 20.27\% larger than those in Case 3. In addition, Case 4 under the provided dispatch strategy can promote the participation of pumped storage and BESS in the power dispatch to respond to the uncertainties caused by the renewable power and load. However, it can be seen that the thermal operation cost in Case 4 is $0.02 \%$ larger than that in Case 3. In Case 4, the time interval division results and the time-series characteristics of net load have increased the operation constraints of thermal generators and at the same optimization conditions, which make the optimization feasible region with the objective of thermal generation cost less than that in Case 3. Thus, the optimization result of thermal generation is $0.02 \%$ larger than that in Case 3 . The results with the IEEE39 system under 96-time-interval power dispatch have shown that the power dispatch strategy provided can ensure the operation economy, reduce the ramping operation of thermal generators, and promote the interaction among renewable power energy, pumped storage, and BES.

\section{Conclusions}

In this paper, a time-interval-varying optimal power dispatch method based on the time-series characteristics of net loads is proposed. In order to achieve zero wind curtailment, which means the maximum utilization of wind power, the wind power has priority to be consumed by conventional loads. Their differences, called net load, are the response objectives. A provincial power grid in China is taken as an example; the conclusions follow:

(1) A time-interval-varying power dispatch method is proposed, in which the net load time-series characteristics are analyzed by the RMT and the time intervals are divided adaptively. The number and length of the time intervals are determined by net loads accordingly.

(2) A net load time-series characteristic quantification method is proposed. The net loads have different characteristics in each time interval. The EMD and SampEn are combined to analyze the fluctuation and complexity of the net load subsequence. A comprehensive characteristic quantification index integrated with the numerical characteristic and complexities is provided. The maximum index can reflect the fluctuation and complexity of the net loads, which can help with the data-mode fusion in the dispatch mode determination.

(3) A time-interval-varying power dispatch method is developed to maximize the utilization of the wind power. According to the time division results and quantification characteristics in each time interval, the operation mode of thermal generators and energy storage systems are determined.

(4) An actual provincial power grid in northeast China is used to conduct the proposed method. In Case 2, time is divided into five intervals. The operation mode of thermal generators is optimized to "6-3-8-2-5" operation mode. There are three time intervals that can realize steady operation during which the thermal generators have constant power outputs. Steady operation lasts for $19 \mathrm{~h}$. Benefit from the operation mode is determined based on the quantification index, the reduction of the ramping operation of the thermal generators, and the decrease of relative costs. Because of the steady operation of thermal generators, the interaction between energy storage and wind power are improved, which is beneficial to resource utilization.

(5) In Case 2, the total operation cost, thermal operation cost, and ramping operation cost are all reduced. The environmental cost of $\mathrm{CO}_{2}, \mathrm{NO}_{X}, \mathrm{SO}_{2}$, and smoke dust emissions is decreased. Further, in Case 2, there are 9.06 fewer tons of carbon dioxide and 9.06 more carbon credits than those in Case 1, which certify the environmental friendliness of the proposed method and strategy. 


\begin{abstract}
Author Contributions: Conceptualization, S.H. and H.S.; data curation, J.W.; formal analysis, J.W. and L.Z.; funding acquisition, J.L. (Jinsong Liu); investigation, Z.G. and Y.G.; methodology, S.H.; resources, L.Z. and J.L. (Jinsong Liu); software, J.L. (Jiajue Li); supervision, H.S.; validation, Y.G. and J.L. (Jinsong Liu); visualization, J.L. (Jiajue Li); writing—original draft, S.H.; writing—review and editing, Z.G. and H.S. All authors have read and agreed to the published version of the manuscript.
\end{abstract}

Funding: This work was supported in part by National Key R\&D Program of China (NO.2019YFB150 5400); Science and Technology Project State Grid Corporation of China (5400-202040495A-0-0-00); Science \& Technology Project State Grid Corporation of China (4000-202128061A-0-0-00).

Conflicts of Interest: The authors declare no conflict of interest.

\title{
References
}

1. National Energy Administration [EB/OL]. “14th Five-Year" Power Planning Work Starting. 2020-01-16. Available online: http:/ / www.nea.gov.cn/2020-01/16/c_138709471.htm (accessed on 3 December 2020).

2. Tsai, C.H.; Figueroa-Acevedo, A.; Boese, M.; Mohan, N.; Okullo, J.; Heath, B.; Bakke, J. Challenges of planning for high renewable futures: Experience in the U.S. midcontinent electricity market. Renew. Sustain. Energy Rev. 2020, 131, 109992. [CrossRef]

3. Shariatmadar, K.; Arrigo, A.; Vallée, F.; Hallez, H.; Vandevelde, L.; Moens, D. Day-Ahead Energy and Reserve Dispatch Problem under Non-Probabilistic Uncertainty. Energies 2021, 14, 1016. [CrossRef]

4. Li, J.H.; Wang, S.; Liu, Y.; Fang, J.K. A coordinated dispatch method with pumped-storage and battery-storage for compensating the variation of wind power. Prot. Control Mod. Power Syst. 2018, 3, 21-34. [CrossRef]

5. Wang, W.; Xu, J.; Zhao, X.; Yuan, X.J.; Li, Z.X. Analysis on Peak Load Regulation Status Quo for Coal-fired Power Plants in China. South. Energy Constr. 2017, 4, 18-24.

6. Power Plant Boiler “Burst Tube” Reason. [EB/OL]. Polaris Power Grid. 2020-06-11. Available online: https://www.sohu.com/a/ 401269905_752692?_trans_=010001_grzy (accessed on 1 December 2020).

7. Liu, Y.; Tan, Q.; Han, J.; Guo, M. Energy-Water-Carbon Nexus Optimization for the Path of Achieving Carbon Emission Peak in China Considering Multiple Uncertainties: A Case Study in Inner Mongolia. Energies 2021, 14, 1067. [CrossRef]

8. What Is Carbon Neutral? What Are the Status of Enterprises under the Carbon Neutral Vision? [EB/OL]. Polaris Electricity Selling Power Grid, 2020-11-20. Available online: http:/ / shoudian.bjx.com.cn/html/20201120/1116972.shtml (accessed on 6 December 2020).

9. Eser, P.; Singh, A.; Chokani, N.; Abhari, R.S. Effect of increased renewables generation on operation of thermal power plants. Appl. Energy 2016, 164, 723-732. [CrossRef]

10. Wang, H.C.; Qin, H.; Zhou, C.; Li, F.; Xu, X.H.; Pan, X. Cross-regional Day-ahead to Intra-day Scheduling Model Considering Forecasting Uncertainty of Renewable Energy. Autom. Electr. Power Syst. 2019, 43, 60-72.

11. Zhao, D.M.; Song, Y.; Wang, Y.L.; Yin, J.F.; Xu, C.L. Coordinated Scheduling Model with Multiple Time Scales Considering Response Uncertainty of Flexible Load. Autom. Electr. Power Syst. 2019, 43, 21-32.

12. Galvan, E.; Gutierrez, A.G.; Gonzalez, N. Two-phase short-term scheduling approach with intermittent renewable energy resources and demand response. IEEE Lat. Am. Trans. 2015, 13, 181-187. [CrossRef]

13. Achilles, S.; Schramm, S.; Bebic, J. Transmission System Performance Analysis for High-Penetration Photovoltaics; Office of Scientific \& Technical Information Technical Reports; National Renewable Energy Laboratory: Golden, CO, USA, 2011.

14. Hu, S.B.; Gao, Z.N.; He, H.; Cao, W.P.; Zhao, Y.T.; Zhou, W.; Gu, H.; Sun, H. Adaptive time division power dispatch based on numerical characteristics of net loads. Energy 2020, 205, 118026. [CrossRef]

15. Wang, Y.; Gu, Y.; Ding, Z.; Li, S.N.; Wan, Y.; Hu, X.R. Charging Demand Forecasting of Electric Vehicle Based on Empirical Mode Decomposition-Fuzzy Entropy and Ensemble Learning. Autom. Electr. Power Syst. 2020, 44, 114-124.

16. Fisch, D.; Gruber, T.; Sick, B. Rule: Mining comprehensible classification rules for time series analysis. IEEE Trans. Knowl. Data Eng. 2011, 23, 774-787. [CrossRef]

17. Carpinone, A.; Giorgio, M.; Langella, R.; Testa, A. Markov chain modeling for very-short-term wind power forecasting. Electr. Power Syst. Res. 2015, 122, 152-158. [CrossRef]

18. Sun, M.; Li, J.; Gao, C.X.; Han, D. Identifying regime shifts in the US electricity market based on price fluctuations. Appl. Energy 2017, 194, 658-666. [CrossRef]

19. Qiu, R.C.; Antonik, P. Smart Grid using Big Data Analytics: A Random Matrix Theory Approach.[S.I.]; John Wiley\&Sons Ltd: New York, NY, USA, 2017.

20. Wu, Q.; Zhang, D.X.; Liu, D.W.; Liu, W.; Deng, C.Y. A method for power system steady stability situation assessment based on random matrix theory. Proc. CSE E 2016, 36, 5414-5420.

21. Liu, W.; Zhang, D.X.; Wang, X.Y.; Liu, D.W.; Wu, Q. Power System Transient Stability Analysis Based on Random Matrix Theory. Proc. CSEE 2016, 36, 4854-4863.

22. Chen, W.B.; Chen, Y.P.; Yao, W.; Wen, J.Y. A Random Matrix Theory-based Approach to Fault Time Determination and Fault Area Location. Proc. CSEE 2018, 38, 1655-1664.

23. Xu, X.Y.; He, X.; Ai, Q.; Cai, C.M. A correlation analysis method for operation status of distribution network based on random matrix theory. Power Syst. Technol. 2016, 40, 781-790. 
24. Yan, Y.J.; Sheng, G.H.; Wang, H.; Liu, Y.D.; Chen, Y.F.; Jiang, X.C.; Guo, Z.H. The Key State Assessment Method of Power Transmission Equipment Using Big Data Analyzing Model Based on Large Dimensional Random Matrix. Proc. CSEE 2016, 36, 435-445.

25. Benaych-Georges, F.; Rochet, J. Outliers in the single ring theorem. Probaility Theory Relat. Fields 2015, 165, 1-51. [CrossRef]

26. Richman, J.S.; Moonmann, J.R. Physiological time-series analysis using approximate entropy and sample entropy. Am. J. Physiol. -Heart Circ. Physiol. 2000, 278, H2039-H2049. [CrossRef] [PubMed]

27. Lake, D.E.; Richman, J.S.; Griffin, M.P.; Moorman, J.R. Sample entropy analysis of neonatal heart rate variability. Am. J. Physiol. -Endocrinol. Metab. 2002, 283, 789-797. [CrossRef]

28. Wang, J.H.; Shahidehpour, M.; Li, Z.Y. Security-constrained unit commitment with volatile wind power generation. IEEE Trans. Power Syst. 2008, 23, 1319-1327. [CrossRef]

29. Pan, I.; Das, S. Fractional Order AGC for Distributed Energy Resources Using Robust Optimization. IEEE Trans. Smart Grid 2016, 7, 2175-2186. [CrossRef]

30. Ross Sheldon, M. A First Course in Probability; Machine Press: Beijing, China, 2014.

31. Kotur, D.; Durisic, Z. Optimal spatial and temporal demand side management in a power system comprising renewable energy sources. Renew. Energy 2017, 108, 533-547. [CrossRef]

32. Zhang., S.; Shao, C.; Xiao, W. Research on Red Wine Quality Based on Data Visualization. In Proceedings of the 2020 3rd International Conference on Artificial Intelligence and Big Data (ICAIBD), Chengdu, China, 28-31 May 2020; pp. 128-132.

33. Kennedy, J.; Eberhar, R. Particle swarm optimization. In Proceedings of the ICNN'95-International Conference on Neural Networks, Perth, WA, Australia , 27 November-1 December 1995; IEEE: Piscataway, NJ, USA, 1995. 